Abstracts from the

\title{
Abstracts from the NIH Office of Research on Women's Health 2020 Annual BIRCWH Meeting: Building Interdisciplinary Research \\ Careers in Women's Health December 14, 2020
}

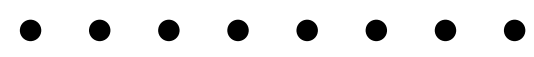




\title{
Building Interdisciplinary Research Careers in Women's Health (BIRCWH)

\author{
Introduction to the Scientific Abstracts, the $20^{\text {th }}$ Anniversary \\ of the BIRCWH Program, and the 2020 Annual BIRCWH Meeting
}

\author{
Lisa Begg, PhD, RN
}

This year, 2020, represents the $20^{\text {th }}$ anniversary of the establishment of the Building Interdisciplinary Research Careers in Women's Health (BIRCWH) program. The BIRCWH Program was created by the Office of Research on Women's Health (ORWH), National Institutes of Health (NIH) and several NIH institutes and centers in the Fall 2000. The program utilizes an institutional mentored research and career development grant program that has increased the number and skills of junior faculty called Scholars who recently completed clinical training or postdoctoral fellowships.

Over 750 women and men have received training through the BIRCWH program since 2000. There are twenty-two BIRCWH programs that are currently funded across seventeen states representing all regions of the United States, with five states each having two programs.

The BIRCWH program supports the ORWH mission and advances its goal to promote the independent scientific careers for a diverse, and robust workforce to advance science for the health of women, and where appropriate, the use of both sexes to better understand the influence of sex as a variable on health and disease.

The BIRCWH Program is built around three pillars: interdisciplinary research, mentoring, and career development. Interdisciplinary science teams work to advance fundamental understanding and solve problems in a manner that a single discipline cannot. The interdisciplinary research can be basic, translational, behavioral, clinical, and/or health-services research relevant to the health of women. Most BIRCWH Scholars move on to obtain independent NIH grant funding following their participation in the BIRCWH Program.

Interdisciplinary mentoring teams are essential to the BIRCWH Program, with a team-based approach for mentoring BIRCWH Scholars. These teams include mentors from diverse disciplines to carry out interdisciplinary projects. Team members may include individuals from medical, dentistry, pharmacy, nursing, veterinary medicine, biotechnology, social sciences, anthropology, genetics, and other disciplines representing different perspectives and areas of expertise. These teams come together to collaborate as a unit, with the common goal of supporting a BIRCWH Scholar in the transition from trainee to independent researcher.

The 2020 Annual BIRCWH meeting will be held virtually on December 14, 2020. There will be a plenary session in the morning, with a special Innovation Lecture to celebrate the $20^{\text {th }}$ anniversary of the BIRCWH program, and a virtual poster session in the afternoon. The published abstracts below will form the basis of the virtual poster session. More detailed information about this meeting and the BIRCWH program can be found on the ORWH website: www.nih.gov/women

Dr. Begg is the Senior Research Program Officer in the NIH Office of Research on Women's Health, overseeing the BIRCWH Program. For further information about the BIRCWH Program and the 2020 Annual BIRCWH Meeting, please contact Dr. Begg at:beggl@od.nih.gov 


\section{BIRCWH Poster Abstracts}

\section{Brigham and Women's Hospital, Harvard Medical School}

\section{Recurrent Episodic Cortical Spreading Depressions Produce Trigeminal Mechanical Allodynia and Anxiety-Related Behaviors}

David Y. Chung, ${ }^{1}$ Aylin Uner, ${ }^{5}$ Andreia Morais, ${ }^{1}$ Tsubasa Takizawa, ${ }^{1,6}$ Tao Qin, ${ }^{1}$ Cenk Ayata, ${ }^{1,2,4}$ Andrea M. Harriott (presenting author) ${ }^{1-4}$

${ }^{1}$ Neurovascular Research Laboratory, Department of Radiology, Massachusetts General Hospital; ${ }^{2}$ Vascular Division, Department of Neurology, Massachusetts General Hospital; ${ }^{3}$ Headache and Neuropathic Pain Division, Department of Neurology, Massachusetts General Hospital; ${ }^{4}$ Harvard Medical School; ${ }^{5}$ Baskent University Faculty of Medicine; ${ }^{6}$ Department of Neurology, Keio University School of Medicine

Background: Cortical spreading depression (CSD) is an intense depolarization underlying migraine aura and implicated in headache. Despite the weight of evidence suggesting CSD is linked to the pain phase of migraine, there remains controversy over a causal role of CSD in cephalalgia, mostly because of the invasive nature of previous CSD methods.

Objective: We used a less invasive optogenetic CSD method to examine the relationships among CSD, trigeminal pain, affective behavior, and cognitive behavior.

Methods: We examined the effect of single and repeated optogenetic CSD (opto-CSD) on mechanical periorbital allodynia using von Frey monofilaments, on spontaneous discomfort using mouse grimace scores, on anxiety behavior using open field testing, and on visuospatial working memory using Y maze testing in male and female mice.

Results: Single-event opto-CSD produced bilateral periorbital mechanical allodynia when tested 1 hour after CSD. The allodynia reversed by 2 days after single opto-CSD. Sumatriptan reversed single opto-CSD-induced periorbital allodynia. In contrast, repeated opto-CSD produced a longer-lasting bilateral periorbital allodynia observed 2 and 4 days after the last CSD event, which reversed when tested at 14 days. Though singleevent opto-CSD produced no change in mouse grimace score, repeated opto-CSD increased mouse grimace scores. Repeated opto-CSD also produced increases in thigmotaxis score, a measure of anxiety. Neither single nor repeated opto-CSD affected visuospatial working memory. There were no significant sex differences in pain, anxiety, or cognitive phenotypes.

Conclusions: These data support a direct relationship between trigeminal pain and anxiety-like behavior and most likely reflect CSD modulation of hypothalamic, thalamic, or neurolimbic pain pathways.

\section{The Impact of Makorin Ring Finger Protein 3 on Sex Differences in Delayed Puberty}

Lydie Naule, ${ }^{1}$ Soukayna Chouman, ${ }^{1}$ Marciana Johnson, ${ }^{1}$ Margaret Henning, ${ }^{1}$ Rona S. Carroll, ${ }^{1}$ Ursula B. Kaiser, ${ }^{1}$ Stephanie A. Roberts (presenting author) ${ }^{1,2}$

${ }^{1}$ Division of Endocrinology, Diabetes and Hypertension, Brigham and Women's Hospital; ${ }^{2}$ Division of Endocrinology, Boston Children's Hospital

Background: Delayed puberty affects $2 \%$ of adolescents. Boys are at increased risk of delayed puberty; however, nonfunctional etiologies of delayed puberty in girls are less understood.

Objectives: Loss-of-function mutations in $M K R N 3$, an inhibitor of pubertal onset, result in central precocious puberty; however, the potential for increased $M K R N 3$ expression to delay pubertal onset has not been reported.

Methods: Wild-type female and male C57BL/6 mice were injected intracerebroventricularly on postnatal day (PND) 1 or by stereotaxic injection in the hypothalamic arcuate nucleus as adults (PND90-120) with a recombinant adeno-associated virus (rAAV-MKRN3-IRES-EGFP) to overexpress $M K R N 3$ or with a control virus (rAAV-EGFP).

Results: Female neonatal mice injected with rAAV-MKRN3IRES-EGFP had significantly delayed puberty by vaginal opening and first estrus $(P<0.005)$ with normal subsequent estrous cyclity and fertility, whereas neonatally injected male mice exhibited normal puberty onset by preputial seperation. In addition to increased MKRN3 expression, mRNA levels of KISS1, $T a c 2$, and $P D Y N$ - expressed in the mediobasal hypothalamus, an area important for pubertal onset-were significantly increased. MKRN3-overexpressing postpubertal male mice exhibited lower basal luteinizing hormone values and impaired response to senktide but not kisspeptin; the impact on postpubertal females is currently being analyzed.

Conclusions: Neonatal overexpression of MKRN3 results in delayed puberty in female mice through effects downstream of KISS1 expression, suggesting that altered MKRN3 regulation may be an unrecognized cause of delayed puberty, especially in girls. $M K R N 3$ overexpression can also lead to hypogonadism in postpubertal mice, suggesting $M K R N 3$ could be a future therapeutic target in the treatment of precocious puberty and other reproductive disorders. 


\section{Duke University}

3. Recruitment Recommendations for Studies Using GPS to Measure Physical Activity Locations Among Hispanic/Latina Women in a Community Health Clinic Setting

Daniela Sotres-Alvarez, ${ }^{2}$ Lauren Hart, ${ }^{1}$ Dori Steinberg, ${ }^{3,4}$ Truls Østbye, ${ }^{1,4}$ Dianne S. Ward, ${ }^{5}$ Katelyn M. Holliday (presenting author) ${ }^{1}$

${ }^{1}$ Department of Family Medicine and Community Health, Duke University; ${ }^{2}$ Deprtment of Biostatistics, University of North Carolina at Chapel Hill; ${ }^{3}$ Department of Nursing, Duke University; ${ }^{4}$ Duke Global Health Institute, Duke University; ${ }^{5}$ Department of Nutrition, University of North Carolina at Chapel Hill

Background: Using Global Positioning System (GPS) devices to record physical activity locations provides important formative data for multilevel physical activity interventions. However, previous studies have reported mixed success recruiting Hispanic/Latino populations for GPS-based studies.

Objective: To qualitatively explore the willingness of Hispanic/ Latina women attending a local community health clinic to enroll in a study using GPS to gather physical activity location data and to assess their recommendations for recruitment.

Methods: The Duke Clinical and Translational Science Institute (CTSI) facilitated a focus group with 9 women recruited from the clinic using the Vanderbilt Community Engagement Studio Toolkit. Study staff introduced the study in Spanish, and CTSI staff led a qualitative inquiry into the group's questions, concerns, and suggestions for recruitment.

Results: Women (ages 24-40; 50\% < high school education, $50 \%$ high school diploma or equivalent) were most concerned about ensuring that the GPS did not transmit data to study staff in real time. They said new arrivals to the United States might be worried about immigration, but they were all interested and willing to participate in the study. They suggested using peer recruitment, showing individuals the GPS devices during recruitment, translating all materials into Spanish, and including simple directions for using the devices.

Conclusions: Hispanic/Latina women attending a local community health clinic were willing to join a study using GPS to gather formative data on physical activity locations. They also provided recommendations to facilitate recruitment of Hispanic/ Latina women for studies using GPS.

\section{Questions as a Marker of Communication Deviance After Right Hemisphere Brain Damage}

Adam Jacks, ${ }^{3}$ Davida Fromm, ${ }^{4}$ Eugene Oddone, ${ }^{5}$ Jamila M. Minga (presenting author) $)^{1,2}$

${ }^{1}$ Communication Disorders Program, North Carolina Central University; ${ }^{2}$ Department of Obstetrics and Gynecology, Duke University; ${ }^{3}$ Department of Allied Health Sciences, University of North Carolina at Chapel Hill; ${ }^{4}$ Department of Psychology, Carnegie Mellon University; ${ }^{5}$ Department of Veterans Affairs, Duke University

Background: Challenges with information gathering after right hemisphere brain damage (RHD) contribute to failed communication. Few studies have examined questions, an explicit way to gather information, as a contributor to communication impairment.
Objective: To determine whether adults with chronic RHD and healthy controls (HCs) differed in the frequency and type of questions produced during 2 tasks.

Methods: Adults with RHD (conversation: $\mathrm{n}=15,52 \pm 9.5$ years; object task: $n=29,52 \pm 12.39$ years; $48 \%$ female) and HCs (conversation: $\mathrm{n}=15,45 \pm 11$ years; object task: $\mathrm{n}=21$, $49 \pm 9$ years; $81 \%$ female) participated. Questions were elicited during recorded face-to-face video sessions in which participants (1) had a 5-minute "first encounter" conversation and (2) asked questions to determine the purpose of unfamiliar objects (a requested minimum of 3 questions per object). Question productions were orthographically transcribed and coded by type using an established coding scheme (polar [yes/no], content [wh-], or alternative [A or B]). Distributions of questions by type and task were compared.

Results: Adults with RHD asked fewer questions during conversation than HCs $(P=0.03)$. Also, the RHD group used significantly fewer polar questions during both tasks than HCs $(\mathrm{t}[21]=3.5, P=0.002$; object task: $\mathrm{t}[48]=3.4, P=0.001)$, although more polar questions were produced during the object task than during the conversation.

Conclusions: Infrequent conversational question use and overall diminished polar question use during both tasks after RHD suggest a production impairment based on frequency and type. Replicating findings in a larger cohort of survivors would aid in determining whether polar questions are an indicator of impairment that can be targeted for treatment.

\section{Leakage from Accountable Care Organizations Among Gynecologic Cancer Patients: Prevalence, Predictors, and Impact on Annual Medicare Expenditures}

Haley A. Moss (presenting author), ${ }^{1,2}$ Oyomoare Osazuwa-Peters, ${ }^{3}$ Michaela Dinan, ${ }^{1,3}$ Evan Myers, ${ }^{2}$ Laura J. Havrilesky ${ }^{1,2}$

${ }^{1}$ Duke Cancer Institute; ${ }^{2}$ Department of Obstetrics and Gynecology, Duke University; ${ }^{3}$ Department of Population Health, Duke University

Background: Accountable care organizations (ACOs) are a network of doctors and hospitals that assume responsibility for the cost and quality of medical care for a defined population. ACOs have not been shown to be successful in reducing spending or improving outcomes for patients receiving cancer care. We hypothesize that there is a significant leakage, or cancer care outside of the assigned ACO, particularly among gynecologic cancer patients. This leakage of care may contribute to higher cost and less coordination of care.

Objective: To determine the prevalence of leakage and identify patient and ACO factors that predict care outside of the ACO.

Methods: This will be a retrospective cohort study using claims data for all beneficiaries in the Medicare Shared Savings Program and a national 5\% random sample of Medicare beneficiaries from 2012 through 2018. ACO-level characteristics will be obtained from the Centers for Medicare \& Medicaid Services' ACO public-use files pertaining to the Shared Savings Program. We will use Poisson regression models to calculate age-adjusted prevalence of ACO leakage. A multivariable model will be used to determine patient and ACO factors related to leakage.

Conclusion: We theorize that ACO leakage negatively impacts effective care coordination because the ACO remains 
financially responsible for the cost of care but is not able to control costs. Future directions include comparing Medicare expenditures among ACO-assigned patients who receive cancer care from a non-ACO provider versus only ACO providers and evaluating sex differences in the prevalence of leakage in other cancer types.

\section{Emory University}

\section{Differences in Follicular Fluid Cytokine Profile in Women with Diminished Ovarian Reserve}

Sina Abhari, ${ }^{1}$ Heather S. Hipp, ${ }^{1}$ Sabrina Gerkowicz, ${ }^{4}$ Quinton S. Katler, ${ }^{1}$ Laurie J. McKenzie, ${ }^{3}$ Weirong Shang, ${ }^{1}$ Alicia K. Smith, ${ }^{2}$ Anna K. Knight (presenting author) ${ }^{2}$

${ }^{1}$ Department of Gynecology and Obstetrics, Division of Reproductive Endocrinology and Infertility, Emory University; ${ }^{2}$ Department of Gynecology and Obstetrics, Division of Research, Emory University; ${ }^{3}$ Division of Reproductive Endocrinology and Infertility, Baylor College of Medicine; ${ }^{4}$ IVFMD, Boca Raton, Florida

Background: Approximately $30 \%$ of women seeking in vitro fertilization have diminished ovarian reserve (DOR), which makes ovarian stimulation and successful retrieval of viable oocytes more difficult.

Objectives: To compare follicular fluid cytokine concentrations in women with DOR and controls and explore correlated functional pathways.

Methods: Women undergoing ovarian stimulation and oocyte retrieval were recruited from the Emory Reproductive Center. We included 51 women with DOR and 30 controls. Follicular fluid samples were collected from each participant at the time of oocyte retrieval and stored for later use. Samples were then assessed using multiplexed sandwich ELISA-based quantitative array platform (RayBiotech). Quantile regression was used to assess the association of DOR diagnosis and cytokine concentrations.

Results: Out of 357 cytokines, expression of 26 cytokines was found to be significantly different in the follicular fluid of DOR patients than it was in the follicular fluid of controls (FDR $<0.05$ ), after adjustment for age and body mass index. Differentially expressed cytokines belong to diverse functional groups involved in follicular development and maturation, including growth factor and related proteins, receptor signaling, apoptosis, and inflammation.

Conclusion: We found that 26 cytokines expressed differently in women with DOR than they expressed in controls with normal ovarian reserve. Our data suggest feasibility of identifying biomarkers that illuminate how the ovarian follicle microenvironment is altered in the DOR population and identification of pathways playing a role in pathophysiology of DOR.

\section{Risk Factors for Post-traumatic Stress Disorder After Breast Cancer Diagnosis and Treatment Among African American Women: A Longitudinal Study}

Andrew Miller, ${ }^{1}$ Preethi Subhedar, ${ }^{2}$ Jennifer S. Stevens (presenting author) ${ }^{1}$

${ }^{1}$ Department of Psychiatry and Behavioral Sciences, Emory University School of Medicine; ${ }^{2}$ Department of Surgery, Emory University School of Medicine
Background: Post-traumatic stress disorder (PTSD) is known to affect twice as many women as men. Breast cancer diagnosis and treatment are common traumas, affecting 1 in 8 women in the US, and $11-30 \%$ of patients are estimated to develop PTSD. However, little research has addressed PTSD risk among higherrisk groups, including African American women, with high rates of repeated trauma and other forms of chronic stress.

Objective: To investigate risk factors for heightened symptoms of PTSD among African American women diagnosed with breast cancer. We hypothesized that prior stressors-including chronic trauma, financial stressors, and race-related stresswould increase risk for PTSD development.

Methods: There were 24 African American women enrolled at the time of a biopsy visit at a publicly funded hospital with a model program for reducing disparities in breast cancer outcomes. PTSD and depression symptoms were assessed at 3 timepoints over the next 12 months.

Results: Among participants with positive breast cancer findings, 50\% met diagnostic criteria for PTSD 6 weeks later. In contrast, among cancer-free participants matched for trauma history, none met criteria for PTSD. Additionally, pre-biopsy levels of race-related stress $(\mathrm{r}=0.59, P<0.05)$ and cancerrelated stress $(\mathrm{r}=0.71, P<0.05)$ were correlated with 6 -week PTSD symptoms irrespective of cancer diagnosis.

Conclusions: Findings highlight an often-overlooked mental health impact of breast cancer and identify risk factors that differentially affect African American women. Future analyses will incorporate neuroimaging and inflammation assessed prebiopsy, with a goal of developing a prognostic model of PTSD risk.

\section{Differences in Oral Glucose Response Curve Shapes in African American Men and Women Presenting with Ketosis-Prone Diabetes}

Priyathama Vellanki (presenting author), ${ }^{1}$ Lucia Cotton, ${ }^{1}$ Darko Stefanovski, ${ }^{2}$ Omolade Oladejo, ${ }^{1}$ Guillermo Umpierrez ${ }^{1}$

${ }^{1}$ Division of Endocrinology, Metabolism, and Lipids, Emory University School of Medicine; ${ }^{2}$ Department of Biostatistics, University of Pennsylvania School of Veterinary Medicine

Background: Ketosis-prone type 2 diabetes mellitus (KPDM) affects obese African American (AA) patients with new-onset diabetic ketoacidosis (DKA) and has a 3:1 male-to-female prevalence. With intensive insulin treatment, about $70 \%$ achieve near normoglycemia remission (HbA1c $<7 \%$, fasting blood glucose $[\mathrm{BG}]<130 \mathrm{mg} / \mathrm{dl}$, and off insulin for 1 week). The shape of the glucose response curve during an oral glucose tolerance test (OGTT) is predictive of future glycemic failure and beta cell function, with biphasic $(\mathrm{B} \varphi)$ curves showing better glycemic control and beta cell function than monophasic $(\mathrm{M} \varphi)$ and incessant rise (IR) curves.

Objective: To study whether $\mathrm{B} \varphi$ shape at remission is associated with lower hyperglycemia relapse compared with $\mathrm{M} \varphi$ and IR curves in men and women.

Methods: There were 71 AA patients $(n=25$ women, $n=46$ men) who underwent a 2-hour $75 \mathrm{~g}$ OGTT after near normoglycemia remission and at the end of the study. Disposition index, the measure of insulin secretion accounting for insulin sensitivity, was compared among the different curves in men and women. Hyperglycemia relapse-free survival $(B G>180 \mathrm{mg} / \mathrm{dl}$, HbA1c $>7 \%$ ) was calculated with the Kaplan-Meier estimator and compared among the curve shapes in men and women. 
Results: At remission, OGTT did not show a significant difference in curve shape between men and women $(P=0.08)$. In men, disposition index differed significantly among the different curve shapes $(\mathrm{B} \varphi: 1.69 \pm 2.14, \mathrm{M} \varphi: 0.97 \pm 1.25$, IR: $0.29 \pm 0.50$; $P=0.04)$. There were no differences among women $(P=0.64)$. Hyperglycemia relapse-free survival did not differ by curve shape in men or women.

Conclusions: In obese AA patients with KPDM, a biphasic OGTT curve shape is significantly associated with higher insulin secretion in men but not women.

\section{Johns Hopkins University}

\section{Sociocultural Mechanisms to Reproductive Autonomy Among Young Black Women Experiencing Reproductive Coercion in Baltimore, Maryland}

Areisa Peters, ${ }^{2,3}$ Kamila A. Alexander (presenting author) ${ }^{1,2}$

${ }^{1}$ Johns Hopkins University School of Nursing; ${ }^{2}$ Department of Population, Family and Reproductive Health; Johns Hopkins Bloomberg School of Public Health; ${ }^{3}$ Indiana University School of Medicine

Background: Because of legacies of injustice, black women report reproductive coercion (RC) almost 3 times more often than white women, but little is known about sociocultural mechanisms underlying reproductive autonomy.

Objectives: To describe prevalence of RC types and analyze sociocultural mechanisms related to reproductive autonomy among young black women who have experienced RC.

Methods: Drawn from a parent study, women aged 18-25 years completed a tablet-based survey. Those who reported at least one RC experience $(n=57)$ were invited to complete a semistructured interview $(n=17)$ that explored sociocultural mechanisms of RC. We used descriptive statistics to report participant characteristics and RC types. We managed qualitative data in Dedoose 9.4 software. We developed a codebook, used thematic analysis, compared data patterns, and reconciled discrepancies.

Results: Mean age was 21 years $(\mathrm{SD}=2.2)$, and physical $(70.2 \%)$ and sexual $(49 \%)$ violence were prevalent. More than one-third $(40.4 \%)$ reported 2 or more pregnancies, and more than half $(51 \%)$ had sexually transmitted infection (STI) histories. A majority $(82.5 \%)$ experienced pregnancy coercion (e.g., pressure and threats), whereas $61 \%$ reported birth control sabotage (e.g., forced sex without a condom). Sociocultural mechanisms were (1) motherhood as protection and sacrifice; (2) independence \& self-sufficiency through financial stability; (3) self-respect and self-esteem to avoid social stigma; and (4) guilt \& shame derived from RC experiences.

Conclusions: Participants described ways sociocultural mechanisms inform perceptions of RC experiences among black young women. Pregnancy pressure from family members was a new finding. Future research could employ sociocultural safety strategies to design pregnancy and STI interventions serving black women experiencing RC.

10. Factors Associated with Endometrial Hyperplasia or Cancer Symptoms in Overweight and Obese Premenopausal Women

Larry Cheskin, ${ }^{2}$ Anne F. Rositch, ${ }^{3}$ Amanda N. Fader, ${ }^{1}$ Anna L. Beavis (presenting author) ${ }^{1}$
${ }^{1}$ Kelly Gynecologic Oncology Service, Department of Gynecology and Obstetrics, Johns Hopkins University School of Medicine; ${ }^{2}$ Department of Nutrition and Food Studies, College of Health and Human Services, George Mason University; ${ }^{3}$ Johns Hopkins University

Background: Overweight and obese women are at higher risk of endometrial hyperplasia or cancer (EH/EC), and many have abnormal bleeding symptoms associated with EH/EC.

Objective: To identify patient factors associated with abnormal bleeding symptoms in overweight and obese women seeking care at a weight management clinic.

Methods: A retrospective cohort study of women presenting for care at the Johns Hopkins Weight Management Center from July 2018 to May 2019 was conducted. The prevalence of abnormal bleeding among premenopausal women was computed. Poisson regression identified factors associated with abnormal bleeding symptoms, which could represent potential symptoms of EH/EC.

Results: There were 56 women included, with a median age of 41 years (interquartile range [IQR]: $33.5-45.5$ ) and a median body mass index (BMI) of $34.3 \mathrm{~kg} / \mathrm{m}^{2}$ (IQR: $31.8-40.1$ ). Most women were white $(70 \%, \mathrm{n}=36)$ or black $(20 \%, \mathrm{n}=10)$. There was 1 woman $(2 \%)$ who had a history of EH. There were 33 women $(59 \%)$ who reported abnormal bleeding symptoms, most commonly heavy and irregular menses. There were 21 women (38\%) who were using hormonal contraceptives. Most factors were not associated with abnormal bleeding, including age, BMI, race, diabetes, hypertension, infertility, hyperlipidemia, sleep apnea, and gravida. Patients with polycystic ovarian syndrome (PCOS) were more likely to have abnormal bleeding symptoms (RR 1.75; 95\% confidence interval [CI]: 1.34-2.28), whereas women on hormonal contraceptives were almost $50 \%$ less likely (RR 0.52; 95\% CI: 0.29-0.93).

Conclusions: In this at-risk population, symptoms of EH/EC were prevalent, more commonly in women with PCOS. Symptoms were less common in women who were already taking hormonal contraceptives, an intervention known to reduce the risk of $\mathrm{EH} / \mathrm{EC}$ in this population.

\section{Evaluating Ovarian Reserve in Women with Sickle Cell Disease}

Lydia H. Pecker (presenting author), ${ }^{1,3}$ Jacquie Maher, ${ }^{2,3}$ Christina Mitchell, 2,3 Maria Facadio-Anterno, 2,3

Sophie Lanzkron, ${ }^{1}$ Mindy Christianson ${ }^{2,3}$

${ }^{1}$ Division of Hematology, Department of Medicine, Johns Hopkins University School of Medicine; ${ }^{2}$ Division of Reproductive Endocrinology and Infertility, Johns Hopkins University School of Medicine, ${ }^{3}$ Department of Obstetrics and Gynecology, Johns Hopkins University School of Medicine

Background: Reproductive counseling for the growing number of women with sickle cell disease (SCD) is limited by a knowledge gap: Clinicians do not know whether SCD or hydroxyurea, a chemotherapeutic that is the main treatment for $\mathrm{SCD}$, causes accelerated ovarian aging, diminished ovarian reserve (DOR), or premature ovarian failure.

Objectives: This ongoing study's objectives are to measure ovarian reserve in young women with SCD and determine the acceptability of this research among eligible women.

Methods: This cross-sectional study is enrolling women from 18 to 32 years of age with SCD and combines chart review for demographic and SCD-related variables with prospective measures of ovarian reserve, including day 3 anti-müllerian hormone 
$(\mathrm{AMH})$, follicle-stimulating hormone (FSH), and transvaginal or transabdominal ultrasound-measured antral follicle count (AFC). DOR was defined conservatively: $\mathrm{AMH}<1.1 \mathrm{ng} / \mathrm{mL}$ or AFC $<7$. Descriptive analyses were performed.

Results: To date, 30 women have been approached. Of those women, 27 consented and 10 completed testing, with $50 \%$ preferring transabdominal ultrasound to transvaginal ultrasound. Among the 10 completed subjects, mean age was $26.5 \pm 3.0$ years, with AMH $3.11 \pm 1.97 \mathrm{ng} / \mathrm{mL}$, FSH 8.24 $\pm 3.29 \mathrm{IU}$, and AFC $16.6 \pm 12.1$. There were 2 subjects who had DOR, with a mean age of $28.09 \pm 0.97$ years, AMH $0.96 \pm 0.08 \mathrm{ng} / \mathrm{mL}, \mathrm{FSH}$ 11.05 $\pm 1.3 \mathrm{IU}$, AFC 7. Neither had been pregnant. Both had taken hydroxyurea. Subjects without DOR $(\mathrm{n}=8)$ had a mean age of $25.4 \pm 3.3$ years, with AMH $3.64 \pm 1.82 \mathrm{ng} / \mathrm{mL}$, FSH $6.5 \pm 3.3 \mathrm{IU}$, AFC $19 \pm 12.3$. Most had been pregnant $(\mathrm{n}=5)$ and exposed to hydroxyurea $(n=5)$.

Conclusions: Women with SCD are willing to participate in ovarian reserve evaluations. Here, $20 \%$ of women, all under 30 years old, have DOR; further study is warranted and ongoing.

\section{Echocardiographic Parameters Normalize 4 Years After Preeclampsia Diagnosis Despite New-Onset Hypertension}

Anum Minhas, ${ }^{2}$ Garima Sharma, ${ }^{2}$ Theresa Boyer, ${ }^{1}$ Alexia Debrosse, ${ }^{1}$ Pamela Ouyang, ${ }^{2}$ Sammy Zakaria, ${ }^{2}$ Monica Mukherjee, ${ }^{2}$ Arthur J. Vaught (presenting author) ${ }^{1}$

${ }^{1}$ Division of Maternal-Fetal Medicine, Department of Gynecology and Obstetrics, Johns Hopkins University School of Medicine; ${ }^{2}$ Division of Cardiology, Department of Medicine, Johns Hopkins University School of Medicine

Background: Preeclampsia increases lifelong cardiovascular disease (CVD) risk. However, it is unclear whether antecedent subclinical disease is present before clinical/apparent CVD.

Objective: To observe echocardiographic changes in women with preeclampsia at 2 time points: index disease and 4 years later.

Methods: This is a longitudinal cohort study comparing echocardiographic findings of women with preeclampsia with severe features (PEC) versus normotensive controls. PEC was defined by the American College of Obstetricians and Gynecologists' criteria. Participants were from a previous prospective cohort study comparing echocardiographic findings in women with PEC versus controls. Detailed echocardiographic parameters to assess clinical and subclinical disease were performed by American Society of Echocardiography guidelines.

Results: We contacted 10 of 36 controls and 16 of 63 women with PEC from our previous cohort. All women were within 4-5 years of preeclampsia diagnosis. We previously reported there were statistically significant differences in right ventricular systolic pressure, left ventricular (LV) posterior wall thickness, LV septal e', LV septal E, and LV septal E/e' at initial echocardiogram. However, these differences were no longer present by the time of 4-year $( \pm 0.5$ year) follow-up (Table 1$)$. Body mass index (BMI) and race are significantly different between groups, with the preeclampsia group having more black women and higher BMI. However, at follow-up, there was no difference in BMI. At follow-up, women with preeclampsia had a trend toward new-onset hypertension $(53.8 \%$ versus $10 \%, P=0.07)$.

Conclusion: We found a trend toward new-onset hypertension within 4 years; however, echocardiographic abnormalities normalized. Larger longitudinal studies using biomarker, clinical, echocardiographic, and other imaging should be performed.

\section{Magee-Womens Research Institute}

\section{Opportunity for HCV and HIV Screening and Primary Prevention of Reproductive-Aged Women Post-Incarceration}

Ingrid S. Macio, ${ }^{2}$ Danielle A. Litzinger, ${ }^{2}$ Leslie A. Meyn, ${ }^{2}$ Elizabeth E. Krans, ${ }^{1,2}$ Sharon L. Hillier, ${ }^{1,2}$

Catherine A. Chappell (presenting author) ${ }^{1,2}$

${ }^{1}$ Department of Obstetrics, Gynecology and Reproductive Sciences, University of Pittsburgh; ${ }^{2}$ Magee-Womens Research Institute

Background: The injection drug use epidemic resulted in increased hepatitis $\mathrm{C}$ virus (HCV) and HIV prevalence among young people. Jails and halfway houses are underutilized venues to screen for bloodborne pathogens.

Objectives: We conducted a cross-sectional study of recently incarcerated reproductive-aged women to estimate prevalence and correlates of HCV and HIV infection.

Methods: Between May 2019 and January 2020, participants between the ages of 18 and 46 years who were within 1 year of incarceration and residing in a post-incarceration facility were recruited. Participants were tested for HIV and HCV. Risk factors for both HCV and HIV were assessed using a selfadministered electronic survey and compared between those who were HCV antibody-positive and those who were HCV antibody-negative using Fisher's exact test and Student's t-test.

Results: Participants enrolled $(n=81)$ had a mean age of 34 years; $69 \%$ identified as white, $27 \%$ as black, and $4 \%$ as Latino. Though none of the participants was positive for HIV, $45(56 \%)$ were positive for HCV antibodies, and 35 (43\%) had active HCV infection, defined as a detectable HCV RNA. Of those with active HCV infection, 13 (37\%) were new diagnoses. Risk factors for $\mathrm{HCV}$ exposure included white race, injection drug use, treatment for overdose, and a sexual partner with $\mathrm{HCV}(P<0.05)$.

Conclusions: This cohort of recently incarcerated women has a high prevalence of active $\mathrm{HCV}$ infection and is at risk for HIV acquisition. Testing for $\mathrm{HIV}$ and $\mathrm{HCV}$ and linkage to primary HIV/HCV prevention and HCV treatment services should be prioritized among women who are being released from incarceration.

\section{Race Differences in Postpartum Blood Pressure Trajectory}

Lara Lemon, ${ }^{2,3}$ Camila Cabrera, ${ }^{2}$ Amal Javaid, Anna Binstock, ${ }^{2}$ Beth Quinn, ${ }^{2}$ Jacob Larkin, ${ }^{1,2}$ Andrew R. Watson, ${ }^{5}$ Richard H. Beigi, ${ }^{2}$ Hyagriv Simhan, ${ }^{1,2}$ Alisse Hauspurg (presenting author) ${ }^{2}$

${ }^{1}$ Magee-Womens Research Institute; ${ }^{2}$ Department of Obstetrics, Gynecology and Reproductive Sciences, Magee-Womens Hospital; ${ }^{3}$ Department of Clinical Analytics, University of Pittsburgh Medical Center; ${ }^{4}$ University of Pittsburgh School of Medicine; ${ }^{5}$ Department of Surgery, University of Pittsburgh School of Medicine

Background: Maternal morbidity and mortality are increasing in the United States. The majority of cases occur in the postpartum period, and there are significant racial disparities, particularly related to hypertensive disorders of pregnancy (HDPs).

Objectives: To describe the postpartum blood pressure (BP) trajectory in the first 6 weeks postpartum after an HDP, utilizing home BP monitoring, and to determine whether trajectories differ by self-reported race. 
Methods: This is a prospective observational cohort study of deliveries between January 2018 and January 2020 at a single tertiary care center. Women with an HDP were enrolled in a postpartum remote $\mathrm{BP}$ monitoring program at the time of delivery. We used repeated BP measures to fit mixed-effects linear regression models.

Results: There were 1,077 participants included in this analysis. We found that both systolic and diastolic BP decline rapidly in the first 3 weeks postpartum and that there is a significant difference in BP trajectory by race, with black women having a more adverse postpartum BP trajectory than white women. Our findings persisted in multivariable models, with adjustment for pre-pregnancy body mass index, tobacco use, type of HDP, and use of antihypertensive medication.

Conclusions: Compared with white women, black women have a less rapid decline in BP postpartum, which persisted in various sensitivity analyses and resulted in more hypertensionrelated hospital readmissions and higher BP by 6 weeks postpartum. Our findings have potential implications for reducing disparities in postpartum hypertension-related morbidity and mortality. Given the proportion of women with persistent hypertension, our findings also support the need for Medicaid extension beyond 60 days postpartum.

\section{Affective Dimensions of Postpartum Pain Are Predictive of Increased Opioid Requirements}

Grace Lim (presenting author), ${ }^{1,2}$ Kelsea R. LaSorda, ${ }^{1}$ Bedda Rosario, ${ }^{1}$ Elizabeth Krans, ${ }^{2}$ Steve Caritis ${ }^{2}$

${ }^{1}$ Department of Anesthesiology and Perioperative Medicine, University of Pittsburgh; ${ }^{2}$ Department of Obstetrics, Gynecology and Reproductive Sciences; University of Pittsburgh

Background: Sensory, cognitive, and affective pain dimensions influence medication decisions. However, no known studies have assessed pain type associations with hospital opioid use.

Objectives: We investigated postpartum pain type, pain intensity, and opioid dose.

Methods: We retrospectively analyzed postpartum women's pain during hospitalization. Patients described pain using Shortform McGill Pain Questionnaire and PROMIS affective/evaluative, visceral/nociceptive, somatic/nociceptive, dynamic/evoked, neuropathic, nociceptive, and nociceptive and/or neuropathic pain codes. Intensity for each descriptor was scored (0-10). Multivariable mixed-effects analyses assessed between- and withinperson relationships for pain type (primary predictor) and intensity (0-10); and pain type (primary predictor) and oxycodone dosage (mg). Opioid use disorder's (OUD) influence on these relationships was assessed.

Results: There were 2,609 women who provided a total of 44,522 unique pain scores/descriptors. Women with affective pain required $1.04 \mathrm{mg}$ more opioids $(P<0.001)$ than women without affective pain, after controlling for age, gravidity, parity, and delivery mode. Similarly, but to a lesser extent, other pain types were associated with increased opioids. Intensity was nonspecific and associated with multiple pain types. OUD history increased the relationships among pain type, pain intensity, and opioid dose. Times when OUD women had affective pain resulted in pain scores that were 0.93 point higher; among women with OUD, those with affective pain had pain scores that were 6.24 points higher than expected. Similarly, OUD women with nociceptive and/or neuropathic pain had $1.56 \mathrm{mg}$ higher oxycodone doses; among OUD women, oxycodone doses were $11.38 \mathrm{mg}$ higher than expected for women with this pain type.

Conclusions: Pain type is linked to postpartum opioid requirements; affective pain dimensions contributed the most to this relationship. Pain intensity scores $(0-10)$ are inadequate to assess the postpartum acute pain experience.

\section{Mayo Clinic}

\section{Biomechanical Muscle Stiffness Measures of Extensor Digitorum Explain Potential Mechanism of McArdle Sign}

Filippo Savoldi, ${ }^{5}$ Zahra Nasr, ${ }^{5}$ Brian G. Weinshenker, ${ }^{5}$ Nathan D. Schilaty (presenting author) ${ }^{1-4}$

${ }^{1}$ Department of Orthopedic Surgery, Mayo Clinic; ${ }^{2}$ Sports Medicine Center, Mayo Clinic; ${ }^{3}$ Department of Physiology and Biomedical Engineering, Mayo Clinic; ${ }^{4}$ Department of Physical Medicine and Rehabilitation, Mayo Clinic; ${ }^{5}$ Department of Neurology, Mayo Clinic

Background: McArdle sign is a phenomenon of impaired gait and muscle weakness that occurs with neck flexion, immediately reversible with neck extension. A recent report demonstrated the specificity of this sign for multiple sclerosis (MS), with differences of peak torque of the extensor digitorum between neck extension and flexion.

Objective: To demonstrate muscle stiffness and associated neuromechanical error as further criteria to evaluate MS.

Methods: This substudy included 73 participants (29 MS, 20 other myelopathies [OM], 19 controls [CON], 5 peripheral nerve lesion [PNL]; age 47.9 years [12.5]). Participants performed 5 extensor digitorum isoinertial contractions with the neck positioned in flexion or extension. Torque and displacement data were post-processed to extract a linear fit of stiffness during the epoch of active finger extension torque. Additional neuromechanical error variables extracted were R-squared and the sum of squared error of prediction (SSE).

Results: Peak torque between extension and flexion was greater in MS patients than in CON $(P<0.001)$, OM $(P<0.001)$, and PNL $(P=0.004)$. MS patients also had greater neuromechanical error compared with $\mathrm{CON}(P=0.023)$ and $\mathrm{OM}$ $(P=0.003)$. Predicative variables ( $\Delta$ peak torque, $\triangle$ SSE, SSE in extension) demonstrated an AUC of 0.95 comparing MS with OM, with overall $80 \%$ specificity and $97 \%$ sensitivity.

Conclusions: The sensitivity-specificity balance for McArdle sign utilizing muscle stiffness is superior to that previously reported $(A U C=0.84)$. Adding variables of neuromechanical error increased the specificity/sensitivity for distinguishing MS from OM, PNL, and CON. Neuromechanical error is most likely caused by a conduction block between the cerebellum and the motor neurons because of demyelination, which is exacerbated by neck flexion.

\section{Prospective Echocardiographic Findings of Global and Regional Left Ventricular Function in Acute Spontaneous Coronary Artery Dissection}

Marysia S. Tweet (presenting author), ${ }^{1}$ Patricia A. Pellikka, ${ }^{1}$ Jae K. Oh, ${ }^{1}$ Sharonne N. Hayes ${ }^{1}$

${ }^{1}$ Department of Cardiology, Mayo Clinic 
Background: Spontaneous coronary artery dissection (SCAD) is a nonatherosclerotic etiology of acute coronary syndrome predominantly affecting women.

Objective: To assess global and regional left ventricular function by echocardiography during and after acute SCAD.

Methods: Patients diagnosed with acute SCAD in Rochester, Minnesota, from January 5, 2016, to March 12, 2020, consented for a study echocardiogram performed during their hospitalization.

Results: There were 34 SCAD patients who were imaged. The median age was 55 years (range 33-63), with most patients being female $(n=32,94 \%)$ and white $(n=31,91 \%)$. The majority $(n=23,68 \%)$ presented with non-ST-elevation myocardial infarction; $11(32 \%)$ presented with ST-elevation myocardial infarction; and $3(9 \%)$ had cardiac arrest. The left anterior descending coronary artery was most commonly affected $(n=20$, $59 \%)$. There were $11(32 \%)$ patients who were hypertensive during echocardiography, and 21 (62\%) had fibromuscular dysplasia. Mean left ventricular ejection fraction (LVEF) was $58 \pm 8 \%$ (range $40-70)$. There were $24(71 \%)$ patients who had regional wall motion abnormalities (RWMA), 23 of whom had left ventricular apex RWMA without thrombus. There were $12(36 \%)$ patients who had apical akinesis ( $\geq 2$ segments). Of the 21 patients with follow-up study echocardiograms at a median of 5 months, the LVEF was $\geq 50 \%$ in all; however, 5 (23\%) had persistent akinesis of the left ventricular apex. Two patients had another SCAD during the study.

Conclusions: While most patients had preserved global systolic function during acute SCAD, many had RWMA and akinesis of the left ventricular apex that persisted on repeat imaging. It will be important to monitor for complications that can be associated with these findings, such as ventricular arrhythmias and apical thrombus.

\section{Medical University of South Carolina}

\section{Brief Computerized Approach Bias Modification Intervention Can Reduce Alcohol Use and Sexual Risk Behavior Among Women with History of Rape}

Christine K. Hahn, ${ }^{1}$ Raluca M. Simons, ${ }^{2}$ Jeffrey S. Simons, ${ }^{2}$ Austin M. Hahn (presenting author) ${ }^{1,2}$

${ }^{1}$ Department of Psychiatry and Behavioral Sciences, Medical University of South Carolina; ${ }^{2}$ Department of Psychology, University of South Dakota

Background: Interventions that reduce alcohol use and risky sexual behavior among women who have experienced rape are urgently needed.

Objective: To examine whether an approach bias modification (ABM) intervention would be effective in reducing alcohol use and increasing condom use among high-risk women with a history of rape.

Methods: This is secondary analyses from a proof-ofprinciple study evaluating the effectiveness of a brief computerized $\mathrm{ABM}$ to reduce alcohol use and increase condom use among high-risk college students $(n=102)$. The current study examined the intervention among 62 high-risk women between the ages of 18 and $24(M=19.76 ; S D=1.41)$. Participants were randomly assigned to either a training condition or a shamcontrol condition. Among the participants, 13 women who reported experiencing rape were in the treatment condition, and 9 were in the control condition. Among those who did not report experiencing rape, 20 were in each condition. Participants in the training condition were trained to make avoidance movements away from alcohol stimuli and approach movements toward condom stimuli over 4 training sessions.

Results: Among those in the training condition, biases changed in the expected direction, regardless of rape history. Similarly, there was a significant increase in condom use among those in the training condition, regardless of rape history $[\mathrm{F}(1,119)=4.14, P=0.044]$. Women who reported a history of rape and were randomized to the treatment condition showed the strongest reductions in alcohol use between baseline and a 3-month follow-up $[\mathrm{F}(1,115)=5.53, P=0.020]$.

Conclusions: $\mathrm{ABM}$ intervention reduced alcohol use and increased condom use among high-risk college women who had experienced rape.

\section{Sex Differences in Arc Regulation and Behavioral Relevance in the Nucleus Accumbens}

Dalia Martinez, ${ }^{1}$ Brandon Hughes, ${ }^{1}$ Christopher W. Cowan, ${ }^{1}$ Rachel D. Penrod (presenting author) ${ }^{1}$

\section{${ }^{1}$ Department of Neuroscience, Medical University of South Carolina}

Background and objective: The nucleus accumbens (NAc) is a key brain region mediating reward, motivation, and moodrelated behaviors. Sex differences are observed in numerous behaviors relevant to NAc function, including anxiety-like and drug-related behaviors. Despite the importance of NAc function and the observed sex differences in relevant behaviors, we have little understanding of the molecular mediators of these differences. Recent work from our laboratory has identified activityregulated cytoskeleton-associated protein (Arc) in the NAc as a potential mediator of sex differences in NAc-relevant behavior.

Methods: To examine Arc induction following behavioral experience, NAc samples from adult wild-type male and female mice were collected following gentle handling, elevated plus maze (EPM) exposure, or cued fear conditioning, and mRNA was isolated for qRT-PCR analysis. To examine the behavioral relevance of Arc in the NAc, male and female wild-type mice received intra-NAc infusions of Arc shRNA (shArc) or control shRNA (shCntrl). Following recovery and Arc knockdown, mice were assessed for anxiety-like and drug-related behaviors.

Results: We found that males induce Arc in the NAc following EPM exposure and that loss of Arc decreases anxietylike behavior. In contrast, females fail to induce Arc following EPM exposure and do not show changes in anxiety-like behavior. Interestingly, Arc knockdown also reduced cocainerelated behavior only in males.

Conclusions: These findings demonstrate sex differences in the induction and requirement for Arc in the NAc. Further studies on sex differences in Arc regulation and action may provide novel insights into neurobiological mechanisms of sex differences in behavior.

\section{Gender Differences in Cannabis Coping Motives Among Emerging Adults: Moderating Effect of Total Lifetime Cannabis Use Exposures}

Aimee L. McRae-Clark, ${ }^{1}$ Jenny Nankoua, ${ }^{1,2}$ Jade Tuttle, ${ }^{1}$ Kevin M. Gray, ${ }^{1}$ Rachel L. Tomko (presenting author) ${ }^{1}$

${ }^{1}$ Department of Psychiatry and Behavioral Sciences, Medical University of South Carolina; ${ }^{2}$ College of Charleston 
Background: Gender differences in negative affect-related cannabis use among adolescents/emerging adults have been mixed. Some research suggests that female adolescents/emerging adults endorse more cannabis coping motives and have more cooccurring mental health concerns than males, whereas others find no gender differences. Given that females have shown faster progression from occasional to problematic use and negative affect increases with the progression of substance use disorder, these mixed results about gender differences may be caused by failure to account for total lifetime cannabis exposures. At early stages of use, gender differences may be minimal. We hypothesized that females would report greater use of cannabis to cope with fewer lifetime cannabis exposures relative to males, consistent with females' faster progression to problematic use.

Objective: To examine whether gender differences in cannabis coping motives are moderated by lifetime exposure to cannabis use.

Methods: Emerging adults (18-21) who averaged at least 3 cannabis use episodes per week $(n=40 ; 55 \%$ female) completed questionnaires detailing total number of lifetime cannabis use sessions, motives for cannabis use (including coping), and symptoms of cannabis use disorder, depression, and anxiety.

Results: Males and females did not differ in reported coping motives. However, gender and total lifetime cannabis exposures significantly interacted to predict coping motives. Coping motives increased for males but not females as lifetime cannabis use episodes increased.

Conclusions: Increasing use of cannabis to cope with increasing lifetime exposures was observed only for males. Females' coping motives either are independent of total cannabis exposure or occur very early in cannabis use onset.

\section{Oregon Health \& Science University}

\section{Gender Differences in Physical Frailty Phenotypes Among Adults with Heart Failure}

Mary Roberts Davis, ${ }^{1}$ Quin E. Denfeld (presenting author) ${ }^{1}$

${ }^{1}$ Oregon Health \& Science University School of Nursing

Background: Physical frailty, a geriatric condition of decreased physiological reserves and increased vulnerability to stressors, is highly prevalent among adults with heart failure (HF). The influence of gender on physical frailty phenotypes in $\mathrm{HF}$, however, is poorly understood.

Objective: To examine gender differences in physical frailty phenotypes among adults with moderate to advanced HF.

Methods: We completed a secondary analysis of data from a study of physical frailty among adults with moderate to advanced HF. Physical frailty was measured with the frailty phenotype criteria, which include unintentional weight loss, weakness, slowness, physical exhaustion, and low physical activity. Those meeting 3 or more criteria were considered physically frail. Comparative statistics and logistic regression were used to analyze gender differences in physical frailty phenotypes.

Results: The average age of the sample $(n=49)$ was $57.4 \pm 9.7$ years; $33 \%$ were female; $67 \%$ had non-ischemic etiology. Women reported significantly worse physical exhaustion than men $(P=0.002)$. There were no significant gender differences in the other criteria. Physical frailty was present in $42.4 \%$ of men and $62.5 \%$ of women, but this was not significantly different $(P=0.19)$. After adjusting for comorbidities and the Seattle Heart Failure Model risk prediction score, women were 3.6 times more likely to be considered physically frail than men (OR $=4.57$ [1.03, 20.36], $P=0.046)$.

Conclusion: Understanding gender differences in physical frailty phenotypes provides clinicians an opportunity to recognize patients at risk for physical frailty, including the associated worse clinical and patient-reported outcomes.

\section{State Medicaid Coverage of Transgender Services Expands Access to Care}

K. John McConnell, ${ }^{2}$ Jae Downing (presenting author) ${ }^{1}$

${ }^{1}$ School of Public Health, Oregon Health \& Science University; ${ }^{2}$ Center for Health Systems Effectiveness, Oregon Health \& Science University

Background: Protections for transgender people in health care remain uncertain in light of the finalized rule on Section 1557 of the Affordable Care Act in June 2020. Less than half of state Medicaid programs cover gender-affirming services. Uncertainty about how paying for gender-affirming services would impact enrollment and health care utilization is a common rationale for denying coverage.

Objectives: To evaluate whether enrollment and health care utilization among transgender people changed in response to coverage of gender-affirming care in Oregon's state Medicaid program in 2015.

Methods: We used a difference-in-differences linear probability model to assess changes in utilization among transgender beneficiaries $(n=5,077)$ before (2010-2014) and after (20152018) the policy, using propensity score-matched cisgender beneficiaries as controls. Diagnoses and procedure and drug codes from Oregon Medicaid claims data were used to identify transgender beneficiaries and outcomes.

Results: After the policy change in 2015, $0.4 \%$ of beneficiaries enrolled were identified as transgender; $56 \%$ of these beneficiaries had filled a prescription for gender-affirming hormones, and $15 \%$ had had a gender-affirming surgery. Coverage of gender-affirming care was also associated with a 21.3percentage-point increase in having a primary care visit among transgender beneficiaries and a 28.3-percentage-point increase in having a mental health visit.

Conclusions: Oregon's coverage of gender-affirming care, while benefiting only a small percentage of beneficiaries, effectively expanded care for transgender people.

\section{Oxytocin Administration in Low-Risk Labor and Birth: Duration of Infusion and Likelihood of Postpartum Hemorrhage}

Nicole S. Carlson, ${ }^{2}$ Elise N. Erickson (presenting author) ${ }^{1}$

${ }^{1}$ Midwifery Division, School of Nursing, Oregon Health \& Science University; ${ }^{2}$ Nell Hodgson Woodruff School of Nursing, Emory University

Background: Postpartum hemorrhage (PPH) contributes to significant maternal morbidity and mortality. The association of uterine stimulation with oxytocin during labor and PPH is unclear.

Objective: To determine whether the duration of oxytocin administration is associated with PPH in a sample of low-risk women. 
Methods: This is a cross-sectional analysis of Consortium on Safe Labor data. After exclusions of high-risk conditions associated with PPH (cesarean operation, hypertension, severe lacerations, etc.), we examined 27,072 vaginal births. PPH was defined as a total blood loss at delivery of more than $500 \mathrm{~mL}$ and/or a diagnostic code for PPH. We included covariates if they were associated with both oxytocin use and PPH and did not mediate oxytocin use. Regression models estimated PPH likelihood overall and within induced and spontaneous labor separately. Subgroup analyses examined oxytocin duration within specific durations of labor.

Results: Overall, 3.9\% $(1,052)$ of women experienced PPH. When oxytocin was used to accelerate spontaneous labor, PPH was 2.17 times as likely (1.59-2.96) when the infusion lasted at least 4 hours compared with an infusion of 2 hours or less. This association was consistent despite longer duration of first- or second-stage labor. During labor induction, oxytocin was associated with higher adjusted odds for PPH when infusion lasted more than 7 hours (OR 1.51, 1.05-2.19) compared with less than 2 hours. The odds specifically increased when the total labor induction lasted more than 12 hours and/or the second stage was more than 3 hours.

Conclusion: Oxytocin use is independently associated with $\mathrm{PPH}$ in many labors. Opportunities to help reduce PPH in otherwise low-risk vaginal birth could address judicious oxytocin administration.

\section{Factors Associated with Less-Than-Biennial Mammogram Screening Behavior Among Oregon Women at Screening Age}

Jeong Youn Lim, ${ }^{2}$ Kenneth Xu, ${ }^{1}$ Paige E. Farris, ${ }^{3}$

Grace Curran, ${ }^{1}$ Jackie Shannon, ${ }^{3}$ Zhenzhen Zhang (presenting author) ${ }^{1}$

${ }^{1}$ Division of Oncological Sciences, Knight Cancer Institute, Oregon Health \& Science University; ${ }^{2}$ Biostatistics Shared Resource, Knight Cancer Institute, Oregon Health \& Science University; ${ }^{3}$ OHSU-PSU School of Public Health, Oregon Health \& Science University

Background: Women with breast cancer diagnosed from mammogram screening have a lower risk of death than women diagnosed with symptoms without mammogram screening. Current screening guidelines recommend routine mammograms starting at age 40, and annual or biennial screening intervals are recommended depending on individual risk. Thus, biennial mammograms are the clinical minimum.

Objectives: To identify factors associated with not having had a mammogram during the previous 2 years to help cancer prevention and early detection efforts.

Methods: This study utilizes area-based probabilistic sampling survey data collected in Oregon in 2019. Weights for the dataset were calculated using a raking approach. Demographic and behavioral information was collected from a cancer-focused questionnaire. The current study sample included women aged 40-89 years. We conducted weighted multivariable logistic regression analyses to identify factors associated with not having had a mammogram during the previous 2 years.

Results: The study included 430 women with a median age of 65 years. Among them, $20.23 \%(n=87)$ reported not having had a mammogram during the previous 2 years, including $3.02 \%$ reporting never having had a mammogram. The adjusted model included the following variables: rural/urban status, age, race, education, marital status, occupation, body mass index, cancer history, insurance status, smoking status, and cancer beliefs. We found that education level $(\mathrm{OR}[95 \% \mathrm{CI}]=3.26[1.65,6.42]$ non-college graduates versus college graduates) and insurance status (OR $[95 \% \mathrm{CI}]=7.42[1.59,34.52]$ uninsured versus insured) were significantly associated with whether women had had a mammogram during the previous 2 years.

Conclusions: Lack of a college degree and of health insurance are associated with less frequent mammogram screening behavior.

\section{Tufts University}

\section{Sex as an Effect Modifier of the Association Between Co-Exposure to Multiple Toxic Metals and the Risk of Type 2 Diabetes}

Emily Riseberg, ${ }^{1}$ Mark Woodin, ${ }^{1,2}$ Rachel Melamed, ${ }^{3}$ Tanya L. Alderete, ${ }^{4}$ Katherine A. James, ${ }^{5}$ Laura Corlin (presenting author) $^{1,2}$

${ }^{1}$ Department of Public Health and Community Medicine, Tufts University School of Medicine; ${ }^{2}$ Department of Civil and Environmental Engineering, Tufts University School of Engineering; ${ }^{3}$ Biomedical Data Science, University of Chicago; ${ }^{4}$ Department of Integrative Physiology, University of Colorado Boulder; ${ }^{5}$ Department of Environmental and Occupational Health, Colorado School of Public Health, University of Colorado Anschutz Medical Campus

Background: It is unknown whether sex modifies associations between coexposure to multiple metals and type 2 diabetes (T2D).

Objective: To assess sex-dependent associations among coexposure to metals, fasting glucose (FG), and T2D incidence.

Methods: Over 1,600 San Luis Valley Diabetes Study participants (54\% female) contributed 1-7 sets of environmental and health measurements over 14 years. We natural logtransformed FG $(\mathrm{mg} / \mathrm{dL})$ and urinary concentrations of metals $(\mu \mathrm{g} / \mathrm{L}$; arsenic [As], cadmium [Cd], manganese [Mn], lead [Pb], uranium [U], and tungsten [W]). We assessed associations between metals coexposure and $\operatorname{lnFG}$ using sex-stratified linear mixed-effect models with a random intercept for each participant. We assessed associations between baseline metals coexposure and diabetes incidence using sex-stratified Fine and Gray competing risk regression models (competing event $=$ all-cause mortality). Based on an evidence-based directed acyclic graph, covariates included age, ethnicity (Hispanic/not), education ( $<12 / 12 />12$ years), smoking status (current/former/never), alcohol intake (grams per week), carbohydrates (grams per day), body mass index, and urine creatine $(\mu \mathrm{g} / \mathrm{g})$.

Results: Sex did not modify the crude diabetes incidence (women $=3 / 100$ person-years; men $=3 / 100$ person-years) or mean time at risk (women $=9.3$ years; men $=9.2$ years). In women, doubling $\ln \mathrm{As}$ and $\ln \mathrm{Pb}$ concentrations was associated with lnFG (\% change $[95 \% \mathrm{CI}]=3[0,5] ;-4[-3,-7]$, respectively). $\mathrm{LnCd}$ and $\operatorname{lnPb}$ were associated with diabetes incidence (subdistribution hazard ratio $[\mathrm{SHR} ; 95 \% \mathrm{CI}]=1.4[1.2,1.8] ; 0.8[0.7$, $0.9]$, respectively). In men, doubling $\ln \mathrm{As}, \ln \mathrm{Mn}, \ln \mathrm{Pb}$, and $\ln \mathrm{U}$ concentrations was associated with $\operatorname{lnFG}(\%$ change $[95 \%$ $\mathrm{CI}]=4[1,7] ;-5[-2,-7] ;-3[-1,-5] ; 4[0,8]$, respectively). LnAs and $\ln U$ were associated with diabetes incidence (SHR $[95 \% \mathrm{CI}]=1.3[1.0,1.5] ; 1.3[1.1,1.5]$, respectively).

Conclusions: Sex modifies the relationship between coexposure to metals and T2D. 


\section{Sex-based Comparison of Lymphocyte Counts Among Male and Female Heart Transplant Recipients}

David R. Snydman, ${ }^{1}$ Jennifer K. Chow, ${ }^{1}$ Whitney A. Perry (presenting author) ${ }^{1}$

${ }^{1}$ Division of Geographic Medicine and Infectious Diseases, Tufts Medical Center

Background: Even though infection is one of the most important complications after solid organ transplantation (SOT), the impact of sex on immune response in this setting has not been well-studied. Immunocompetent men and women have demonstrated quantitative differences in markers of both innate and adaptive immunity, including T-cell lymphocyte subsets. Lymphocytes are of particular interest given the use of immunosuppressive medications that impair T-cell immunity and predispose to severe infection.

Objective: To compare absolute lymphocyte count (ALC) measurements of male and female heart transplant recipients immediately before and 1 month after transplantation.

Methods: Retrospective cohort study examining 375 adult heart transplant recipients from 2000 to 2018 at Tufts Medical Center. ALC measurements and other clinical and demographic variables were collected from the electronic medical record. Mean ALCs collected at both time points were compared between sexes using Student's t-test.

Results: We analyzed 104 female and 271 male recipients. There were 40 subjects ( 15 female, 25 male) who were missing a pre-transplant ALC and were excluded from that portion of the analysis. The mean ALCs for females and males prior to transplantation were $1.6(\mathrm{SD}=0.7)$ and $1.3(\mathrm{SD}=0.6)$, respectively $(P<0.001)$. One month after transplantation, females had a mean ALC of $1.2(\mathrm{SD}=0.9)$ and males had a mean ALC of 0.8 $(\mathrm{SD}=0.6)(P<0.001)$.

Conclusions: Female heart transplant recipients have a higher ALC prior to transplantation, which persists in the setting of iatrogenic impairment of T-cell immunity. Such differences in immune markers may have important implications in how male and female SOT recipients respond to infection and vaccination.

\section{Evaluation of the Sex-Dependent Impact of Prenatal Opioid Exposure on the Developing Brain}

Tomoko Kaneko-Tarui, ${ }^{2}$ Alice M. Graham, ${ }^{3}$ Samuel Carpenter, ${ }^{3}$ Neel Madan, ${ }^{4}$ Tomo Tarui, ${ }^{5}$

Jonathan M. Davis, ${ }^{1}$ Jill L. Maron, ${ }^{1,2}$ Elizabeth Yen (presenting author) ${ }^{1,2}$

${ }^{1}$ Department of Pediatrics, Tufts Medical Center; ${ }^{2}$ Mother Infant Research Institute, Tufts Medical Center; ${ }^{3}$ Department of Psychiatry, Oregon Health \& Science University; ${ }^{4}$ Department of Radiology, Tufts Medical Center

Background: Neonatal abstinence syndrome (NAS) is a public health crisis. About half of exposed infants, predominantly males, develop severe withdrawal and feeding difficulty/ hyperphagia requiring pharmacologic intervention (NAS-Tx). There is an urgent need to better understand the neurobiological mechanisms underlying NAS and to aid in risk stratification and care allocation.

Objective: To elucidate potential neurobiological mechanisms responsible for hyperphagia and severe withdrawal through salivary gene expression analyses and brain magnetic resonance imaging (MRI) (structural MRI [sMRI] and restingstate functional MRI [rsfMRI]).

Methods: Salivary transcriptomic profiles were obtained within 48 hours of life, and the expression of select hypothalamic/ reward genes-NPY2R, LEPR, POMC, DRD2-was correlated with the need for and duration of pharmacologic treatment, length of hospital stay (LOS), and caloric intake in 100 sex- and gestational age-matched neonates with and without NAS. sMRI and rsfMRI were obtained in 6 sex-, exposure-, and age-matched neonates.

Results: DRD2, a key reward gene, expression was significantly higher in males than in females with NAS $(\Delta \mathrm{Ct}$ $10.8 \pm 3.8$ versus $13.9 \pm 3.7, P=0.01)$. In NAS-Tx, expression of $L E P R$, an appetite suppressor, was higher in males than in females $(\Delta \mathrm{Ct} 8.4 \pm 2.5$ versus $12.4 \pm 5.1, P=0.05)$. $D R D 2$ expression significantly correlated with caloric intake at 1 week of life $(\mathrm{r}=0.58, P=0.02)$. Expression of $N P Y 2 R$, an appetite regulator, negatively correlated with $\operatorname{LOS}(\mathrm{r}=-0.24, P=0.05)$. There were 2 females with NAS who had white matter T1 hyperintensity on the sMRI, and 1 male with NAS had a normal sMRI. There were 3 non-exposed neonates who had normal sMRI.

Conclusion: Prenatal opioid exposure exerts sex-dependent effects on hypothalamic feeding-regulating genes. Correlation with sMRI and rsfMRI is ongoing.

\section{Tulane University}

\section{Sex Differences in the Prevalence of Ideal Cardiovascular Health Among US Adults}

Robert C. Hendel ${ }^{2}$ Marie A. Krousel-Wood, ${ }^{1,2}$ Paul K. Whelton, ${ }^{1,2}$ Jiang He, ${ }^{1,2}$ Joshua D. Bundy (presenting author) ${ }^{1}$

\section{${ }^{1}$ Department of Epidemiology, Tulane University School of Public Health and Tropical Medicine; ${ }^{2}$ Department of Medicine, Tulane University School of Medicine}

Background: Although US women may have better cardiovascular health $(\mathrm{CVH})$ than men, underlying reasons for sex differences in $\mathrm{CVH}$ are not well understood.

Objective: To compare the proportions of US women and men with ideal levels of $7 \mathrm{CVH}$ metrics.

Methods: We used data from the National Health and Nutrition Examination Survey (2011-2016) and survey analysis procedures to estimate the proportions of US adults having ideal, intermediate, and poor levels of 7 modifiable American Heart Association CVH metrics. We used logistic regression to evaluate the multivariable-adjusted associations between sex and ideal levels of each CVH metric.

Results: We included 11,696 participants who were 20 years old or older in the analyses. After adjustment for age, race/ ethnicity, education, income level, marital status, and the other $\mathrm{CVH}$ metrics, women had significantly higher odds of nonsmoking (odds ratio [OR], 1.36; 95\% confidence interval [CI], $1.14-1.63$ ), body mass index $<25 \mathrm{~kg} / \mathrm{m}^{2}$ (OR, $1.30 ; 95 \% \mathrm{CI}$, 1.15-1.47), ideal healthy diet score (OR, 2.63; 95\% CI, 1.514.58), and ideal blood pressure levels (OR, 1.96; 95\% CI, 1.702.25) and lower odds of ideal leisure-time physical activity levels (OR, 0.74; 95\% CI, 0.63-0.87) than men. No sex differences were identified in ideal levels of total cholesterol (women versus men OR, 0.95; 95\% CI, 0.82-1.09) or glucose (women versus men OR, 1.12; 95\% CI, 0.99-1.27). 
Conclusions: Compared with US men, US women had significantly higher odds of ideal levels of nonsmoking, body mass index, healthy diet, and blood pressure and lower odds of ideal levels of leisure-time physical activity.

\section{The Influence of Sex, Child Maltreatment, and Loss on Metabolic Syndrome in Adulthood}

Lydia A. Bazzano, ${ }^{2}$ Leia Y. Saltzman (presenting author) ${ }^{1}$

${ }^{1}$ School of Social Work, Tulane University; ${ }^{2}$ Tulane University School of Public Health and Tropical Medicine

Background: Metabolic syndrome is associated with cardiovascular disease, including an increased risk for heart attack, stroke, and death. Child maltreatment and bereavement have also been linked to cardiovascular disease.

Objectives: The primary objective of the study is to test the influence of child maltreatment and bereavement on the risk for metabolic syndrome among adult men and women.

Methods: Stepwise logistic regression was used to estimate the effect of child maltreatment, bereavement, cardiovascular, metabolic, and lifestyle risk factors on the presence or absence of metabolic syndrome as defined by American Heart Association guidelines. Data were drawn from the cross-sectional biomarker subproject of the National Survey of Midlife Development in the United States $(n=1,255)$.

Results: Sex was a significant predictor of risk for metabolic syndrome, such that being female reduced the odds of developing metabolic syndrome by as much as $52 \%(\mathrm{OR}=0.48$, $P<0.001)$. Having a healthy diet and exercising regularly also reduced the risk of developing metabolic syndrome $(\mathrm{OR}=0.73$, $P<0.05 ; \mathrm{OR}=0.73, P<0.05$, respectively). A history of blood pressure or cholesterol problems, as well as being diagnosed with diabetes, increased the risk by as much as $147 \%(\mathrm{OR}=2.13$, $P<0.001 ; \mathrm{OR}=1.66, P<0.01$; and $\mathrm{OR}=2.47, P<0.001)$. Neither child maltreatment nor bereavement was predictive in these models.

Conclusion: Our study found that women were at less risk for developing metabolic syndrome. This is somewhat counter to previous literature suggesting that women are at higher risk than men.

\section{University of California, Davis}

\section{Late-Preterm Dyad Supplementation Practices in Mother-Baby Postpartum Units Across the US}

Valerie J. Flaherman, ${ }^{2}$ Kimberly M. Ngo, ${ }^{1}$ Allison Lloyd-McLennan, ${ }^{3}$ Heather L. Sipsma, ${ }^{4}$ Beth A. King, ${ }^{5}$ Carrie A. Phillipi, ${ }^{6}$ Laura R. Kair (presenting author) ${ }^{1}$

${ }^{1}$ Department of Pediatrics, University of California, Davis; ${ }^{2}$ Department of Pediatrics, University of California, San Francisco (UCSF); ${ }^{3}$ Pediatrics, UCSF Benioff Children's Hospital Oakland; ${ }^{4}$ Public Health, Benedictine University; ${ }^{5}$ Academic Pediatric Association; ${ }^{6}$ Pediatrics, Oregon Health \& Science University

Background: Late-preterm (34-36 $6^{6 / 7}$ weeks) mother-infant dyads discontinue breastfeeding, on average, sooner than term and earlier-preterm dyads.

Objective: To identify variations in supplementation practices among breastfeeding late-preterm dyads.

Methods: Using SPSS for descriptive statistics, we analyzed data from late-preterm mother-infant dyads from a 2017-2018 emailed survey of supplementation practices among US hospitals in the Better Outcomes for Newborns through Research (BORN) Network.

Results: A total of $71 \mathrm{BORN}$ site physician leaders from discrete hospitals in 32 states completed the survey (74\% response rate). Hospitals varied in their thresholds for infant gestational age required for admission to the mother-baby unit or the neonatal intensive care unit (NICU): $4(6 \%)$ no cutoff, 4 (6\%) 37 weeks, $11(16 \%) 36$ weeks, 49 (69\%) 35 weeks, and 2 (4\%) 34 weeks. There were $58(82 \%)$ that recommended universal pumping by mothers for late-preterm infants. Infant weight-loss thresholds for medically indicated supplementation ranged from universal supplementation at 35 weeks at 6 hospitals (8\%) and at 36 weeks at 4 hospitals $(6 \%)$ to tolerating $15 \%$ weight loss before supplementation at 1 hospital. The most frequently used weight-loss threshold was greater than $10 \%$, used at 17 hospitals (24\%), though a total of 28 different strategies were reported. There were 21 hospitals (30\%) that used donor milk, not formula, as the supplement of choice. Use of bottles (which 59\% of hospitals employed at least sometimes) versus alternative feeding modes (syringe, cup, etc.) was highly variable.

Conclusions: Supplementation practices for breastfeeding latepreterm dyads varied widely across US hospitals. Comparativeeffectiveness research is needed to identify optimal strategies to support longer-term breastfeeding success for late-preterm dyads.

\section{Effects of Sugar-Sweetened Beverage Consumption on Insulin Sensitivity in Black Women Compared With White Women}

Candice Allister Price (presenting author), ${ }^{1}$ Valentina Medici, ${ }^{2}$ Kimber L. Stanhope ${ }^{1,3}$

${ }^{1}$ Department of Molecular Biosciences; School of Veterinary Medicine; University of California, Davis; ${ }^{2}$ Division of Gastroenterology and Hepatology; School of Medicine; University of California, Davis; ${ }^{3}$ Department of Nutrition; University of California, Davis

Background: Black women are the highest consumers of sugar-sweetened beverages (SSB) and are one of the highest risk groups for type 2 diabetes (T2D). Just consuming $12 \mathrm{oz}$. of a soft drink per day is associated with a $27 \%$ increased risk for T2D in black women. Ample evidence from clinical studies demonstrates the detrimental effects of SSB on risk factors associated with cardiometabolic disease; however, none of this evidence stems from studies conducted in black women.

Objective: We propose to examine biomarkers of cardiometabolic disease risk in black and white women consuming SSB for 2 weeks and determine whether plasma metabolites correspond to differential metabolic responses between these racial groups.

Methods: Healthy premenopausal black $(n=20)$ and white $(\mathrm{n}=20)$ women (aged $18-48$ years and BMI $\left.25-35 \mathrm{~kg} / \mathrm{m}^{2}\right)$ will be recruited and provided sucrose-sweetened beverages to consume as $25 \%$ of their energy requirement for 2 weeks.

Results: Based on our preliminary data $(n=10)$, we predict that 2 weeks of consuming sucrose-sweetened beverages will result in greater reductions in insulin sensitivity but lower increases in hepatic fat and lipid biomarkers of cardiometabolic disease in black women compared with white women.

Conclusions: This study will be the first to provide experimental evidence as to whether our understanding of mechanisms of SSBs on risk for cardiometabolic disease can be translated to black women. 


\section{University of California, San Francisco}

\section{Accuracy of Self-Assessment of Gestational Age Using Date of Last Menstrual Period}

Katherine Ehrenreich, ${ }^{1}$ Natalie Morris, ${ }^{1}$ M. Antonia Biggs, ${ }^{1}$ Daniel Grossman, ${ }^{1}$ Lauren Ralph (presenting author) ${ }^{1}$

${ }^{1}$ Advancing New Standards in Reproductive Health; Bixby Center for Global Reproductive Health; Department of Obstetrics, Gynecology and Reproductive Sciences; University of California, San Francisco

Background: Accurate assessment of gestational age (GA) is important in pregnancy-related care.

Objective: To examine the accuracy of self-assessment of GA using last menstrual period (LMP) as compared with clinician ultrasound.

Methods: Between October 2019 and March 2020, we surveyed 1,087 people ages 15 and over seeking abortions at 7 facilities across the US. To explore the magnitude and direction of differences between ultrasound and self-assessment, we used the Pearson correlation coefficient to calculate difference scores by subtracting GA estimated using LMP from the GA on ultrasound. In bivariable linear regression models adjusting for clustering by site, we evaluated whether difference scores varied by certainty about the LMP date and duration of pregnancy.

Results: Mean GA on ultrasound was 62 days; $12 \%$ were in the second trimester. Most respondents $(85 \%)$ provided an LMP date. GA based on LMP and GA on ultrasound were strongly and positively correlated with a Pearson correlation coefficient of 0.80 . The average difference between GA on ultrasound and GA based on LMP was -0.16 days and did not differ based on certainty about LMP date $(P=0.31)$. Difference scores varied based on pregnancy length; compared with participants at 8 to 9 weeks gestation (mean difference -1.7 days), those at 6 weeks gestation or less overestimated duration of pregnancy using LMP by a larger margin $(-5.6$ days, $P=0.002)$, and those at 13 weeks or greater underestimated their gestation using LMP by a larger margin (11.1 days, $P=0.001)$.

Conclusions: Self-assessment of GA using LMP among women is highly accurate but, at gestations less than 13 weeks, tends toward overestimating rather than underestimating duration of pregnancy.

\section{Development of an Obstetrics Comorbidity Risk Score for Clinical and Operational Use}

Vincent Liu, ${ }^{1}$ Monique Hedderson, ${ }^{1}$ Mara Greenberg, ${ }^{2}$ Patricia Kipnis, ${ }^{1}$ Gabriel J. Escobar, ${ }^{1}$

Halley Ruppel (presenting author) ${ }^{1}$

${ }^{1}$ Kaiser Permanente Northern California Division of Research; ${ }^{2}$ Kaiser Permanente Northern California Department of Obstetrics and Gynecology, East Bay

Background: Maternal comorbidities are associated with higher risk for adverse obstetric outcomes. Understanding comorbidity risk profiles prior to delivery hospitalization will support early identification of pregnant women at risk for adverse outcomes.

Objective: We sought to develop an obstetrics comorbidity risk score for automated implementation in the electronic health record (EHR) for clinical and operational use.

Methods: We developed the score using a retrospective cohort of 227,405 pregnancies from Kaiser Permanente Northern California between 2010 and 2017. We used a dichotomous composite outcome of adverse obstetric events (e.g., severe preeclampsia, eclampsia, hemorrhage, death). Candidate predictors included maternal age, parity, and maternal diagnoses documented in the year prior to delivery hospitalization, grouped using HCUP Clinical Classifications Software categories. We used a data-driven approach to develop a parsimonious model easily implementable for real-time calculation in the EHR. We used penalized regression (lasso) for variable selection and logistic regression to fit the final model. We used 10-fold cross-validation to estimate error.

Results: An adverse outcome occurred in $3.8 \%$ of deliveries $(\mathrm{n}=8,726)$. Lasso selected 37 of 277 candidate predictors. The final model demonstrated good discrimination (AUC 0.75) and adequate calibration for $<20 \%$ predicted risk upon inspection of calibration plots. The model captured outcomes not captured by common risk factors alone (e.g., diabetes, hypertension).

Conclusions: A model using preadmission maternal comorbidities demonstrated good performance and outperformed existing comorbidity scores. An automated comorbidity risk score will facilitate early identification of women at high risk for adverse outcomes, supporting the American College of Obstetricians and Gynecologists' recommendation for risk-appropriate levels of maternal care.

\section{University of Colorado}

\section{Risk of Stroke-Associated Pneumonia Among Patients with Chronic Inflammatory Disorders}

Paco S. Herson, ${ }^{2}$ Sharon N. Poisson, ${ }^{3}$ Matthew Lippi, ${ }^{1}$ Adit A. Ginde, ${ }^{1}$ Layne Dylla (presenting author) ${ }^{1}$

${ }^{1}$ Department of Emergency Medicine, University of Colorado School of Medicine; ${ }^{2}$ Department of Anesthesiology, University of Colorado School of Medicine; ${ }^{3}$ Department of Neurology, University of Colorado School of Medicine

Background: Stroke-associated pneumonia (SAP) is a leading cause of medical complications after stroke. Certain chronic inflammatory disorders (CIDs) increase the risk of stroke. However, it remains unclear how the associated chronic inflammation and often-associated immunosuppression with CIDs affect the risk of SAP and whether sex alters this risk.

Objective: Evaluate sex-based differences in risk of SAP in CIDs.

Methods: Discharges in the National Inpatient Sample from 2010 to 2012 and 2015 to 2017 diagnosed with an acute ischemic stroke were classified as having ulcerative colitis (UC), Crohn's disease (CD), rheumatoid arthritis (RA), psoriasis (PS), systemic lupus erythematosus (SLE), other CID (ankylosing spondylitis or polymyalgia rheumatica), multiple CIDs, or none. Multivariable logistic regression assessed for associations between CID, sex, and SAP.

Results: Compared with those discharges without CIDs, there was higher risk of SAP with UC (OR 1.40, 95\% CI 1.04-1.88) in 2010-2012 and UC (OR 1.29, 95\% CI 1.00-1.66) and CD (OR 1.33, 95\% CI 1.00-1.84) in 2015-2017. In 2010-2012, RA was associated with reduced risk of SAP (OR 0.84, 95\% CI 0.73-0.98). Females were not at increased risk of SAP in visits with UC (2010-2012: OR 1.20, 95\% CI 0.71-2.03; 2015-2017: OR 1.14, 95\% CI 0.62-2.07), RA (2010-2012: OR 1.07, 95\% CI 0.78-1.47), or CD (2015-2017: OR 1.41, 95\% CI 0.74-2.70).

Conclusion: The risk of SAP varies with specific CIDs but is unaltered by sex. There was temporal variance in the risk of SAP in some CIDs. Further exploration of the use of immunologic agents is needed to fully understand the potential change in risk of SAP among CID patients. 


\section{Markers of Cardiometabolic Health and Body Composition for Transgender Youths Before and After Testosterone}

Natalie J. Nokoff (presenting author), ${ }^{1}$ Megan M. Kelsey, ${ }^{1}$ Kristen J. Nadeau, ${ }^{1}$ Kerrie L. Moreau ${ }^{2,3,4}$

${ }^{1}$ Department of Pediatrics, University of Colorado Anschutz. Medical Campus; ${ }^{6}$ Department of Medicine, University of Colorado Anschutz Medical Campus; ${ }^{3}$ VA Eastern Colorado Health System; ${ }^{4}$ Geriatric Research Education and Clinical Center

Background: About $1.8 \%$ of youths identify as transgender. Over half are transgender male (female sex at birth, male gender identity). Many will start testosterone therapy to induce secondary sex characteristics that align with gender identity.

Objective: Evaluate differences in cardiometabolic healthincluding body composition, insulin sensitivity, and endothelial function - in transgender males at baseline, at 1 month after testosterone therapy, and at 12 months after testosterone therapy.

Methods: Transgender males $(n=13,13.6-16.8$ years $)$ were followed longitudinally prior to and at 1 month and 12 months after testosterone therapy initiation. Participants had fasting morning labs and measurement of body composition (dualenergy x-ray absorptiometry) and endothelial-dependent vasodilation (brachial artery flow-mediated dilation [FMD] using ultrasound). Insulin sensitivity was estimated by the homeostatic model assessment of insulin sensitivity (HOMA-IR). Measures with 2 time points were compared using paired t-tests; those with 3 time points were compared using repeated measures ANOVA.

Results: The percentage of body fat decreased after 12 months of testosterone therapy (baseline, 12 months: $36.2 \pm 6.5$ versus $30.5 \pm 8.1 \%, P=0.001)$ and percentage of lean mass increased $(60.4 \pm 6.1$ versus $66.4 \pm 7.6 \%, P=0.001)$. Leptin decreased after starting testosterone therapy (baseline, 1 month, 12 months: $45.3 \pm 36.9$ versus $28.3 \pm 16.9$ versus $18.6 \pm 30.0$, $P=0.01)$. Sex hormone-binding globulin decreased after testosterone therapy $(47.4 \pm 22.0$ versus $35.3 \pm 17.5$ versus $23.4 \pm$ $9.1 \mathrm{nmol} / \mathrm{L}, P=0.001)$. HOMA-IR decreased after testosterone therapy $(2.1 \pm 1.5$ versus $2.2 \pm 2.4$ versus $1.3 \pm 0.9, P=0.026)$. Brachial artery FMD did not significantly change from baseline to 1 month after testosterone therapy $(10.0 \pm 3.1$ versus $9.7 \pm 3.5$, $P=0.08$ )

Conclusions: Estimated insulin sensitivity improved, body fat decreased, and lean mass increased after testosterone therapy in transgender male youths. Brachial artery FMD did not change. Future work will evaluate differences between those who did not have endogenous puberty blocked and those who did.

\section{High-Fat Diet Negatively Affects the Expression of Repressors of the PI3K/AKT/NF-kB Signaling Pathway in the Ovaries}

Emily M. Elliott, ${ }^{1}$ Natalie M. Hohos, ${ }^{1}$

Malgorzata E. Skaznik-Wikiel (presenting author) ${ }^{1}$

${ }^{1}$ Department of Obstetrics and Gynecology, University of Colorado School of Medicine

Background: Exposure to a high-fat diet (HFD) in female rodents has been shown to cause ovarian dysfunction. HFDs also cause increased ovarian inflammation, which may be involved in the resulting ovarian dysfunction. The PI3K/AKT/NF-kB pathway is proinflammatory and important in ovarian aging; however, little is known about its role in HFD-induced ovarian inflammation and dysfunction.

Objective: To determine changes in the PI3K/AKT/NF-kB pathway in granulosa cells after HFD exposure.

Methods: Female mice that were 5 weeks old were fed either a $60 \%$ HFD $(n=18)$ or standard chow $(n=6)$ for 10 weeks. After 10 weeks, HFD-fed mice were separated into 3 tertiles based on body weight: HFD-lean $(n=6$, HFLn $\leq 26$ g), middle tertile (excluded, 26-30 g), and HFD-obese ( $=6, \mathrm{HFOb}>30 \mathrm{~g})$. Mice were superovulated with 5IU of pregnant mare serum gonadotropin, followed 48 hours later by $5 \mathrm{IU}$ of human chorionic gonadotropin, and granulosa cells were isolated from the ovaries 20 hours later. qRT-PCR was used to determine the expression of several repressors of the PI3K/AKT/NF-kB pathway (CDKN1A, DUSP5, IER3, NR4A1, ZFP36, TNFAIP6). Differences among groups were determined by a one-way ANOVA, followed by Tukey's range test as appropriate.

Results: After 10 weeks of HFD, HFOb mice had lower levels of ovarian DUSP5, NR4A1, ZFP36, and TNFAIP6 than the chow controls $(P<0.05)$. Both HFOb and HFLn had lower levels of ovarian IER3 than chow controls $(P<0.05)$.

Conclusions: HFD causes downregulation of repressors of the PI3K/AKT/NF-kB pathway in the ovaries. These findings suggest that there is a decrease in the inhibition of this proinflammatory pathway, which may lead to increased ovarian inflammation and subsequent ovarian dysfunction.

\section{Sex-Dependent Regulation of Cardiomyocyte Function by Midkine in Pediatric Dilated Cardiomyopathy}

Cortney E. Wilson, ${ }^{1}$ Anis Karimpour-Fard, ${ }^{1}$

Shelley D. Miyamoto, ${ }^{3}$ Brian L. Stauffer, ${ }^{1,2}$

Carmen C. Sucharov, ${ }^{1}$ Kathleen C. Woulfe (presenting author) ${ }^{1}$

${ }^{1}$ Department of Medicine, Division of Cardiology, University of Colorado School of Medicine; ${ }^{2}$ Department of Medicine, Division of Cardiology, Denver Health and Hospital Authority; ${ }^{3}$ Department of Pediatrics, Division of Cardiology, University of Colorado School of Medicine and Children's

Hospital Colorado

Background: Girls with dilated cardiomyopathy (DCM) have worse outcomes than boys, and the mechanisms that lead to these differences are not clear. We have identified a cytokine, midkine (MDK), that is significantly upregulated in the serum from pediatric DCM patients. MDK is significantly higher in girls with DCM requiring heart transplantation than it is in girls with DCM who are stable.

Objective: To determine whether MDK impacts cardiomyocyte function in a sex-specific manner.

Methods: Cardiomyocytes isolated from juvenile male and female rats (JRVMs) were treated with $1 \mu \mathrm{g}$ MDK for 48 hours. Cardiomyocyte function and calcium dynamics were assessed. In addition, RNA sequencing was completed to identify differentially regulated pathways in juvenile male and female cardiomyocytes in response to MDK.

Results: Female JRVMs treated with MDK had higher peak calcium and slower calcium reuptake than vehicle-treated female JRVMs, whereas peak calcium and calcium reuptake were not changed between MDK-treated and vehicle-treated male 
JRVMs. Myofibril calcium sensitivity was decreased in female JRVMs treated with MDK, whereas calcium sensitivity was unchanged in male MDK-treated JRVMs. Genes that were differentially expressed in cardiomyocytes treated with MDK demonstrated a sex-dependent regulation.

Conclusions: This study demonstrates sex-specific differences in cardiomyocyte function in response to MDK. This suggests that elevated circulating MDK in pediatric DCM patients may lead to different cardiac responses in male and female patients. Therefore, elucidating these sex-specific disease mechanisms is critical to defining therapies focused on male and female pediatric DCM patients.

\section{University of Kentucky}

38. Health Disparities in Preterm Birth: Understanding Barriers and Facilitators Related to Transvaginal Ultrasound Cervical Length Surveillance Among Obstetric Providers and Patients in Appalachian Kentucky

Mairead Moloney, ${ }^{2}$ Anna Hansen, ${ }^{1}$ Cynthia Cockerham-Morris, ${ }^{1}$ Jing Li, ${ }^{3}$ Niraj R. Chavan (presenting author) ${ }^{1}$

${ }^{1}$ Division of Maternal Fetal Medicine, Department of Obstetrics \& Gynecology, University of Kentucky College of Medicine; ${ }^{2}$ Department of Sociology, University of Kentucky College of Arts and Sciences; ${ }^{3}$ Center for Health Services Research, University of Kentucky College of Medicine

Background: Preterm birth (PTB) represents an important health disparity across the state of Kentucky. Transvaginal ultrasound (TVU) measurement of cervical length is the best predictor of PTB risk. However, several provider- and patientrelated barriers limit the uptake of TVU in Appalachian Kentucky.

Objective: To understand TVU-related knowledge, attitudes, and practices and identify determinants that impact the widespread adoption of TVU among obstetric providers and their patients.

Methods: Obstetric providers, as well as patients with a history of PTB, were recruited from 3 community-based clinics. A mixed-methods approach was adopted. Guided by the Consolidated Framework for Implementation Research (CFIR), semi-structured interviews were conducted to gather data about knowledge, attitudes, practices, barriers, and facilitators related to implementing TVU in clinical practice. Providers and patients were also asked about strategies to enhance implementation through multilevel interventions. Transcripts were coded using a multistage coding process based in grounded theory.

Results: There were 14 providers and 22 patients interviewed. Providers believed that sociodemographic characteristics, health behaviors, and clinical comorbidities endemic in Appalachia heightened their patients' PTB risk. Participants stressed the need for increasing PTB awareness and TVU-related education for patients. Most commonly reported barriers to TVU included lack of TVU training, lack of patient access to transportation, low patient comfort level, and low patient social support. Suggestions for facilitating TVU included increasing training for providers and sonographers, patient and public education, and patients' family/social support.

Conclusions: Participants identified multiple TVU-related barriers and facilitators. These data will inform the design of a multifaceted implementation strategy targeting disparities in preterm birth across Appalachian Kentucky.

\section{Longitudinal Trajectories of Psychosocial Functioning in Recently Returned Women Veterans}

Karen A. Lawrence (presenting author), ${ }^{1}$ Dawne S. Vogt, ${ }^{2,3}$ Adam J. Dugan, ${ }^{4}$ Shawn Nigam, ${ }^{4}$ Emily M. Slade, ${ }^{4}$ Brian N. Smith ${ }^{2,3}$

${ }^{1}$ College of Social Work, University of Kentucky; ${ }^{2}$ National Center for PTSD Women's Health Sciences Division, VA Boston Healthcare System; ${ }^{3}$ Department of Psychiatry, Boston University School of Medicine; ${ }^{4}$ Department of Biostatistics, College of Public Health, University of Kentucky

Background: Few studies have examined the relationship between post-military mental health and work and family functioning in post-9/11 women veterans.

Objective: To provide a clearer understanding of prospective trajectories and longitudinal relationships among deployment stressors and post-military mental health and psychosocial functioning.

Methods: This secondary data study used US Department of Veterans Affairs data with 3 time points (T1, T2, T3), spanning 7.5 years, and included 554 women veterans. We measured post-traumatic stress disorder (PTSD) severity, depression severity, alcohol use, and functioning in relationship, parental, and work domains, as well as covariates (e.g., warfare exposure and sexual harassment during deployment). Linear mixed models were used to model trajectories of and relationships among these factors.

Results: Mean depression scores decreased over time $(P<0.001)$ but remained clinically significant. PTSD and alcohol misuse scores did not change significantly. Work functioning decreased from $\mathrm{T} 2$ to $\mathrm{T} 3(\mathrm{~B}=-2.289 ; \mathrm{CI}:-4.217,-0.352$; $P=0.021)$. Relationship and parental functioning did not change significantly. Adjusting for covariates, decreased T2-to-T3 work functioning was predicted by increasing T1-to-T2 PTSD $(\mathrm{B}=0.338 ; \quad \mathrm{CI}: 0.154, \quad 0.520 ; \quad P<0.001)$ and depression (B $=0.397$; CI: $0.141,0.652 ; P=0.003)$. Decreased T2-to-T3 relationship functioning was predicted by increasing T1-to-T2 PTSD ( $\mathrm{B}=0.235$; $\mathrm{CI}$ : $0.068,0.402 ; P=0.006)$ and depression (B=0.471; CI: 0.227, 0.716; $P<0.001$ ). Decreased parental functioning was predicted only by increases in PTSD severity (B =0.286; CI: $0.135,0.438 ; P<0.001$ ).

Conclusions: Our findings suggest that treating symptoms of both PTSD and depression may have significant and widespread benefits to women veterans' post-military work and family functioning.

\section{Estrogenic Control of Fluid Intake Is Lost in Middle-Aged and Old Female Rats in Response to Angiotensin II}

Andrea A. Edwards, ${ }^{1}$ Jessica Santollo (presenting author) ${ }^{1}$

${ }^{1}$ Department of Biology, University of Kentucky

Background: Estradiol (E2) dampens fluid intake, which helps defend fluid homeostasis by preventing excessive extracellular fluid volume. This effect has been exclusively studied in young rats but could be altered in older animals because aging influences the neuronal sensitivity to E2.

Objective: We tested the hypothesis that aging influences the fluid intake and depressor effects of E2.

Methods: Young, middle-aged, and old rats were ovariectomized and implanted with a telemetry catheter and a cerebral ventricular cannula. After recovery, rats were treated with either 
oil or estradiol benzoate (EB) once a day for 2 days. On day 3 , rats were treated centrally with angiotensin II. Water and saline intakes were recorded, and blood pressure was monitored for 1 hour. The following week, the protocol was repeated with animals receiving the opposite hormone treatment.

Results: In young rats, EB treatment reduced water and saline intake. Though EB treatment reduced water intake in middleaged rats, this effect was lost in old rats. Furthermore, EB treatment failed to reduce saline intake in middle-aged and old rats. The depressor effect of E2, however, was intact in all age groups. Next, we measured expression of estrogen receptors in brain areas that control fluid balance. In the paraventricular nucleus (PVN), ER $\beta$ and GPERl expression was lower in aged rats than it was in young rats.

Conclusions: These results suggest that the estrogenic control of fluid intake is modified by age, which may be caused by decreased E2 signaling in the PVN. Thus, older animals are not responsive to the protective effect of $\mathrm{E} 2$ on fluid intake.

\section{University of Illinois at Chicago}

\section{The University of Illinois at Chicago BIRCWH Program}

Pauline M. Maki, ${ }^{1}$ Pavitra Kotini-Shah, ${ }^{2}$ Heather Weinreich, ${ }^{3}$ Beatriz Peñalver Bernabé, ${ }^{4}$ Irina Buhimschi ${ }^{5}$

${ }^{1}$ Department of Psychiatry \& Psychology, Neuropsychiatric Institute, College of Medicine, University of Illinois at Chicago; ${ }^{2}$ Department of Emergency Medicine, College of Medicine, University of Illinois at Chicago; ${ }^{3}$ Department of Otolaryngology, College of Medicine, University of Illinois at Chicago; ${ }^{4}$ Richard and Loan Hill Department of Bioengineering, University of Illinois at Chicago;

${ }^{5}$ Department of Obstetrics \& Gynecology, College of Medicine, University of Illinois at Chicago

Background: The University of Illinois at Chicago (UIC) has a long track record of commitment to women's health research, facilitated by the breadth of basic, translational, and clinical science on our campus. With 21 BIRCWH Scholars to date, we are highly committed to fostering the careers of female (100\% to date) and underrepresented minority (33\% to date) Scholars.

Objective: To promote training and career development for a new generation of physician-scientists and researchers and equip them with the knowledge and career skills necessary to advance science for the health of women in the next decade and beyond. We aim to promote skills to address the barriers faced by women and minority Scholars to foster success in a competitive academic environment.

Approach/Scholars: The 2020-2025 UIC BIRCWH program will train 6 junior MD and/or PhD faculty in women's or sex/gender-based health research. We apply evidence-based mentoring practices to promote team science and leadership development. A key feature of our program is the vertical (Scholars-mentors) and horizontal (inter-Scholar) networking facilitated by our world-class mentors and collaborative culture. Our new Scholars are Pavitra Kotini-Shah, MD, Assistant Professor of Emergency Medicine (cardiac arrest); Beatriz Peñalver Bernabé, PhD, Assistant Professor of Bioengineering (systems biology); and Heather Weinreich, MD, Assistant Professor of Otolaryngology (surgical instrumentation).

Innovation: We provide training on novel research and data analysis approaches to incorporate sex as a biological variable into Scholar research.
Conclusions: The UIC BIRCWH program is strongly aligned with the Trans-NIH Strategic Plan for Women's Health Research in training a diverse workforce able to advance science for the health benefit of women.

\section{University of Minnesota}

\section{Movement, Function, and Lymphedema in Women With and Without Axillary Web Syndrome: 5-Year Follow-Up}

David Hunter, ${ }^{3}$ Anne Blaes, ${ }^{2,4}$ Tufia Haddad, ${ }^{5}$ Linda Koehler (presenting author) ${ }^{1,2}$

${ }^{1}$ Divisions of Physical Therapy and Rehabilitation Science, Department of Rehabilitation Medicine, University of Minnesota Medical School; ${ }^{2}$ Masonic Cancer Center; ${ }^{3}$ Department of Radiology, University of Minnesota Medical School; ${ }^{4}$ Division of Hematology, Oncology and Transplantation; University of Minnesota Medical School; ${ }^{5}$ Department of Oncology, Mayo Clinic

Background: Axillary web syndrome (AWS) is a condition that involves the development of a tight band of tissue in the axilla following breast cancer surgery with lymph node removal. The long-term physical issues associated with AWS are unknown.

Objectives: To determine the long-term physical issues associated with AWS.

Methods: There were 36 women who enrolled in this 5-year longitudinal, observational study, with visits at 2, 4, 12, and 78 weeks and 5 years after surgery. Women were diagnosed with AWS if AWS was identified on a physical exam at any time point within 5 years of surgery. An independent Student's t-test compared goniometric shoulder range of motion; upper extremity function using the Disabilities of the Arm, Shoulder, and Hand (DASH) questionnaire; and lymphedema measures using limb volume, bioimpedance spectroscopy (BIS), and trunk tissue dielectric constant (TDC) in women with and without AWS.

Results: Of the 27 women who completed the 5-year followup visit, $14(52 \%)$ had lingering signs of AWS. Women with AWS had significantly lower abduction passive $(P=0.037)$ and active $(P=0.047)$ range of motion than women without AWS. Active and passive flexion range of motion were lower in the AWS group but did not reach a statistical difference $(P=0.142$, $P=0.223$, respectively). There was no statistical difference in upper-extremity DASH scores $(P=0.667)$ or lymphedema measures (BIS: $P=0.585$, arm volume: $P=0.783$, chest TDC: $P=0.290)$ between the groups.

Conclusion: AWS is a chronic condition in breast cancer survivors and is associated with long-term reduced shoulder motion.

\section{The Impact of Paternal Caregiver Factors on Family Functioning and Youth Adjustment in War-Affected Families}

Maria M. Vukovich, ${ }^{2}$ Joseph E. Gaugler, ${ }^{3}$ Abigail H. Gewirtz, ${ }^{4}$ Cheryl L. Robertson, ${ }^{1}$ Jayne A. Fulkerson, ${ }^{1}$ Sarah J. Hoffman (presenting author) $^{1}$

${ }^{1}$ School of Nursing, University of Minnesota; ${ }^{2}$ University of Denver; ${ }^{3}$ School of Public Health, University of Minnesota; ${ }^{4}$ Department of Family Social Science, University of Minnesota 
Background: Research of war-affected families has more commonly focused on the maternal-child relationship. Evidence describing the impact of paternal caregivers' health and trauma histories on family functioning and youth adjustment is less prevalent in the literature. Yet these are important constructs to consider in the health of the family system.

Objective: To describe associations among paternal-reported family functioning, paternal torture and war trauma exposure, paternal mental and physical health, and youth-reported adjustment in a cohort of Karen immigrant families.

Methods: There were 49 paternal caregivers and 72 youths aged 11-23 years who self-reported using instruments such as the FACES IV questionnaire (family functioning), the Strengths and Difficulties Questionnaire (SDQ) (youth adjustment), and an open-ended assessment of physical health. Paternal caregivers completed the Karen Mental Health Screener (mental health distress) and the General War Trauma and Torture Scale (torture/war trauma exposure) assessments. Multiple regression analyses were conducted to examine associations.

Results: Paternal-reported torture experiences were significantly and positively related to paternal mental health distress and physical health problems $(\mathrm{r}=0.311, P=0.015)$. Paternalreported mental health distress and physical health problems were significantly and negatively associated with youth-reported adjustment ( $\mathrm{r}=0.421, P=0.009)$. Torture experiences in paternal caregivers were found to have a statistically significant and negative association with paternal-reported family functioning $(\mathrm{r}=0.347, P=0.012)$.

Conclusions: Study findings contribute to a better understanding of the distinctive impact of paternal mental health, physical health, and trauma histories on youth adjustment and family functioning. Results will inform the development of interventions for caregivers and youths aimed at improving the well-being of war-affected families.

\section{University of North Carolina at Chapel Hill}

\section{Memantine for Prevention of Cognitive Decline in Patients with Breast Cancer}

Allison M. Deal, ${ }^{2}$ Laura J. Quillen, ${ }^{1}$ Erin A. O'Hare, ${ }^{2}$ Tim A. Ahles, ${ }^{3}$ Eliza M. Park, ${ }^{1,2,4}$ Hyman B. Muss, ${ }^{2,4}$ Zev M. Nakamura (presenting author) $)^{1,2}$

${ }^{1}$ Department of Psychiatry, University of North Carolina at Chapel Hill; ${ }^{2}$ Lineberger Comprehensive Cancer Center, University of North Carolina at Chapel Hill; ${ }^{3}$ Department of Psychiatry and Behavioral Sciences, Memorial Sloan Kettering Cancer Center; ${ }^{4}$ Department of Medicine, University of North Carolina at Chapel Hill

Background: Up to $75 \%$ of patients with cancer report cognitive decline during treatment. There is no established way to treat or prevent cognitive decline caused by chemotherapy. Interventions that are cost-effective, are available to all cancer patients, and can be easily incorporated into oncology care are needed.

Objective: Determine whether memantine can mitigate chemotherapy-related cognitive decline in patients with breast cancer.

Methods: In this single-arm phase 2 study, we will recruit 56 patients with stage 1-3 breast cancer, perform cognitive assessments prior to and 4 weeks after chemotherapy, and administer $10 \mathrm{mg}$ of memantine twice daily during chemotherapy.
Cognitive changes - as measured by a computerized cognitive test (delayed matching-to-sample test, primary outcome), a standard neuropsychological battery, and patient self-reportingwill be compared with those of a historical control group of breast cancer patients undergoing chemotherapy who did not receive memantine.

Results: We have enrolled 20 participants through the first 6 months of our recruitment period. Prior to chemotherapy, $25 \%$ reported at least mild symptoms of cognitive impairment. Compared with controls, participants demonstrated better processing speed (Trail Making Test-Part A, 25.7 versus 41.7 seconds, $P<0.0001)$ and performance on 1 measure of verbal memory (HVLT-R delayed recall, 10.1 versus $8.9, P<0.0001$ ) prior to chemotherapy. Otherwise, no baseline cognitive differences were noted. By the time of the meeting, we will have recruited 35 participants and prepared analysis on the neuroprotective effects of memantine in the first 25 participants.

Conclusions: This study will provide preliminary data on the efficacy of memantine to attenuate chemotherapy-related cognitive decline.

\section{Sex Differences in Peritraumatic CD4+ $T$ Lymphocytes Predict Chronic Pain Severity Following Motor Vehicle Collision Among Black Americans}

Sarah Linnstaedt, ${ }^{1}$ Samuel A. McLean, ${ }^{1}$ Matthew C. Mauck (presenting author) ${ }^{1}$

${ }^{1}$ Institute for Trauma Recovery, Department of Anesthesiology, University of North Carolina at Chapel Hill

Background and Objective: In the US, more than 10 million motor vehicle collisions (MVCs) occur annually and disproportionately affect black American (BA) females. CD4+ T lymphocytes have been implicated in sex differences in pain pathogenesis; however, their role in chronic pain development in BAs following MVC is unknown.

Methods: We performed a retrospective analysis of a prospective, multicenter, emergency department (ED)-based longitudinal cohort study of BAs presenting following MVC. Pain severity, severity of life threat, and RNA samples (PAXgene) were collected at ED enrollment. Pain severity was assessed at 6 weeks, 6 months, and 1 year. Blood RNA sequencing was performed, and proportions of blood CD4+ T lymphocytes to all immune cell phenotypes were estimated via CIBERSORT. Repeated measures linear regression analyses were used to estimate the association between circulating peritraumatic $\mathrm{T}$ lymphocytes and chronic pain severity and determine sex interaction in this association.

Results: We analyzed 183 participants, 63 males and 120 females. After adjustment for age, study site, income, education, and perceived life threat, relative proportion of peritraumatic CD4+ T lymphocytes predicted chronic pain severity $(\beta=6.3$, 95\% CI [2.3-10.4]). A sex by CD4+ T lymphocyte interaction was observed $(\beta=12.0$ [1.6-22.5]); in stratified analyses, the association of peritraumatic $\mathrm{T}$ lymphocytes and chronic pain was significant in females but not males $(\beta=8.3$ [3.1-13.6] versus $\beta=-3.7[-13.0-5.6])$.

Conclusions: Female BAs with increased peritraumatic CD4+ T lymphocytes have worse pain outcomes after MVC. The CD4+ T lymphocyte subset may be responsible for sensitizing nociceptors leading to sex differences and racial differences in chronic pain pathogenesis. 


\section{Sex- and Age-Specific Anatomy of Cerebrospinal Fluid in the Infant Brain}

Mahmoud Mostapha, ${ }^{4,5}$ Martin A. Styner, ${ }^{1,2,4,5}$ Joseph Piven, ${ }^{1,2,5}$ Mark D. Shen (presenting author) ${ }^{1,2,3,5}$

${ }^{1}$ Carolina Institute for Developmental Disabilities, University of North Carolina School of Medicine; ${ }^{2}$ Department of Psychiatry, University of North Carolina School of Medicine; ${ }^{3}$ Neuroscience Center, University of North Carolina School of Medicine; ${ }^{4}$ Department of Computer Science, University of North Carolina at Chapel Hill; ${ }^{5}$ Infant Brain Imaging Study (IBIS) Network

Background: Our previous studies demonstrated that extraaxial cerebrospinal fluid (EA-CSF) surrounding the brain is a promising biomarker for early detection of neurodevelopmental disorders (NDDs) such as autism. The extant literature has been limited to a single, global measure of EA-CSF for the entire brain because a method to detect regionally specific EA-CSF was unavailable.

Objective: For the first time, we developed a method for highdimensional anatomical mapping of EA-CSF, with the goal of detecting sex-, age-, and anatomically-specific differences in early, normative CSF physiology.

Methods: Anatomically precise measures of EA-CSF were measured at 80,000 points across the brain in 153 (96 male and 57 female) typically developing infants with longitudinal magnetic resonance imaging (MRI) scans at 6, 12, and 24 months of age. This method is integrated into a freely available, opensource, cross-platform, user-friendly software package, enabling any neuroimaging lab to quantify EA-CSF in its pediatric studies. Users are invited to contribute their results into a datasharing consortium to test the sensitivity and specificity of EACSF as a biomarker across multiple NDDs.

Results: Specific regions (constituting $6.2 \%$ of the brain) showed significant sex differences, with female infants having lower EA-CSF than males (corrected $P<0.01$ ). Further, specific regions showed a significant sex-by-age interaction, whereby females had more stable EA-CSF between 6 and 24 months $(P<0.01)$.

Conclusions: Sex differences in EA-CSF were located in dorsal midline regions, where CSF drains and clears the brain of neuroinflammation. Discovering sex differences in normal CSF physiology has important implications for NDDs, several of which exhibit impaired CSF physiology and sex differences in neurological and behavioral symptoms.

\section{University of Pennsylvania}

\section{Misinformation About HPV Vaccine on Twitter: An Exploratory Content Analysis}

Mary Putt, ${ }^{2}$ Lyndsay Siegle, ${ }^{3}$ Joseph Cappella, ${ }^{4}$

Raina Merchant, ${ }^{5}$ Alison Buttenheim, ${ }^{6}$

Graciela Gonzalez-Hernandez, ${ }^{7}$

Melanie L. Kornides (presenting author) ${ }^{1}$

${ }^{1}$ School of Nursing; Department of Pediatrics, Perelman School of Medicine; Leonard Davis Institute of Health Economics; Center for Health Incentives and Behavioral Economics; University of Pennsylvania; ${ }^{2}$ Division of Biostatistics; Department of Biostatistics, Epidemiology and Informatics; Perelman School of Medicine; University of Pennsylvania; ${ }^{3}$ Department of Bioengineering, University of Pennsylvania;

${ }^{4}$ Annenberg School for Communication, University of
Pennsylvania; ${ }^{5}$ Perelman School of Medicine; Penn Center for Digital Health; University of Pennsylvania; ${ }^{6}$ School of Nursing; Perelman School of Medicine; Leonard Davis Institute of Health Economics; Center for Health Incentives and Behavioral Economics; University of Pennsylvania; ${ }^{7}$ School of Biostatistics, Epidemiology and Informatics; IBI Health Language Processing Center; Perelman School of Medicine; University of Pennsylvania

Background: Misinformation about the vaccine for human papillomavirus (HPV) on social media may contribute to vaccine hesitancy and impede uptake of this cancer prevention vaccine.

Objective: To identify content and frequency of misinformation about HPV vaccine in text-based data extracted from Twitter.

Methods: Using a retrospective review from December 2019 through March 2020, we retrieved 5,342 unique Englishlanguage tweets, excluding retweets, and randomly selected 4,258 associated with the keyword "\#hpv" for manual annotation. Tweets were categorized as (1) contains misinformation; (2) supports/provides education; or (3) neither supports nor contains misinformation. We compared audience engagement metrics across categories. The content of tweets containing misinformation was further annotated.

Results: Of the 4,258 tweets, $20 \%$ contained misinformation, whereas $65 \%$ provided support/education. The most prevalent categories of misinformation were (1) adverse health effects $(56 \%),(2)$ mandatory vaccination (19\%), and (3) ineffectiveness of the vaccine $(13 \%)$. Among tweets containing health misinformation, nonspecific harm or injury $(41 \%)$, death $(21 \%)$, and that the vaccine causes cervical cancer $(12 \%)$ were most frequently mentioned. Almost one-fifth (19\%) of the tweets containing misinformation mentioned females, whereas only $6 \%$ mentioned males. The majority $(72 \%)$ did not mention gender. On average, tweets conveying concern were retweeted 17 times, whereas those expressing support were retweeted far less frequently, at 7.8 times $(P<0.001)$.

Conclusions: Approximately 20\% of unique "\#hpv" tweets posted in a 3-month period on Twitter contained misinformation about HPV vaccine. Our analysis describes the content and frequency of this misinformation, highlighting the most important targets for corrective interventions.

\section{Characteristics Associated with Postpartum Physical Activity Levels Among Women with Hypertensive Disorders of Pregnancy}

Samantha Murphy, ${ }^{2}$ Michal Elovitz, ${ }^{2}$ Lisa Levine, ${ }^{2}$ Jennifer Lewey (presenting author) ${ }^{1}$

${ }^{1}$ Division of Cardiovascular Medicine, Perelman School of Medicine, University of Pennsylvania; ${ }^{2}$ Maternal and Child Health Research Center and Department of Obstetrics and Gynecology, Perelman School of Medicine, University of Pennsylvania

Background: Hypertensive disorders of pregnancy (HDPs), such as preeclampsia, are associated with increased risk of cardiovascular disease. Postpartum interventions may mitigate this risk but have not been well studied in women with an HDP. STEP UP Mom is a randomized trial to increase physical activity (PA) in postpartum women with an HDP using remote monitoring and social incentives.

Objective: To describe patient characteristics associated with daily step count prior to study randomization. 
Methods: Between October 2019 and May 2020, 168 women were enrolled. We used univariate and multivariable linear regression to identify characteristics associated with step count, as measured by wearable devices. We also compared women with low step count (defined as $\leq 4,800$ steps per day, the lowest step count quartile) with women who took more than 4,800 steps per day, using a chi-square test. This analysis includes 137 participants with complete baseline data available at the end of enrollment.

Results: Mean daily baseline step count was 7,444 ( $\mathrm{SD}=3,453)$. In multivariable regression, characteristics positively associated with baseline step count were good self-reported health $(2,789$ steps, $P<0.001)$, tobacco use $(2,622$ steps, $P=0.03)$ high school education or less $(1,676$ steps, $P=0.019)$, and weeks since delivery (293 steps per week, $P=0.011)$. Participants with low baseline step count $(\leq 4,800$ steps per day) were more likely to enroll after the COVID-19 pandemic (34\% versus $18 \%$, $P=0.04)$ and be married (71\% versus $49 \%, P=0.029)$ compared with those with normal or high step count.

Conclusions: Multiple patient characteristics impact postpartum PA levels, which may be helpful in planning lifestyle interventions in high-risk postpartum women.

\section{University of Texas Medical Branch}

\section{Female Sex Associated with Later-Age Onset of Childhood Obesity}

Claire B. Cummins, ${ }^{1}$ Efstathia Polychronopoulou, ${ }^{2}$ Enshuo Hsu, ${ }^{2}$ Andrea M. Glaser, ${ }^{3}$ Kanika A. Bowen-Jallow (presenting author) ${ }^{1}$

${ }^{1}$ Department of Surgery, University of Texas Medical Branch; ${ }^{2}$ Office of Biostatistics, University of Texas Medical Branch;

${ }^{3}$ Department of Pediatrics, University of Texas Medical Branch

Background: Management of obesity is frequently unsuccessful, and though most interventions have been aimed at school-age children, recent evidence suggests that intervention may be more effective at earlier ages.

Objective: To define the relationship between key familial and sociodemographic factors and the age of onset of childhood obesity.

Methods: All children born at our institution between January 2004 and November 2017 who had their body mass index (BMI) measured between the ages of 3 and 12 years were included. The initial age of obesity was defined as the age at the first encounter where a child had a BMI $\geq$ the $95^{\text {th }}$ percentile. We modeled the time from birth to obesity using Cox proportional hazards models.

Results: A total of 5,723 children were included. Of those, $1,290(22.5 \%)$ were obese and 4,433 $(77.5 \%)$ were non-obese. In all, 2,847 (49.8\%) children were female, and 739 (12.9\%) identified as black, 3,397 (59.4\%) as Hispanic, 1,323 (23.1\%) as white, and $264(4.6 \%)$ as "other". Earlier age of onset of obesity was significantly associated with higher birthweight $(P<0.0001)$, higher maternal BMI $(P<0.0001)$, Hispanic race $(P=0.0087)$, and the presence of maternal postpartum depression $(P=0.043)$. Later age of onset of obesity was significantly associated with female sex $(P=0.047)$, being married $(P=0.037)$, and having an unknown marital status $(P=0.0006)$.

Conclusion: Earlier onset of obesity was significantly associated with higher birthweight, higher maternal BMI, Hispanic race, and the presence of maternal postpartum depression, whereas later age of onset of obesity was significantly associated with female sex, being married, and having an unknown marital status.

\section{Associations Between Maternal Characteristics and Childhood Influenza Vaccination in the National Immunization Survey-Child}

Abbey B. Berenson, ${ }^{1}$ Erika L. Fuchs (presenting author) ${ }^{1,2}$

${ }^{1}$ Center for Interdisciplinary Research in Women's Health, Department of Obstetrics and Gynecology, University of Texas Medical Branch at Galveston; ${ }^{2}$ Sealy Institute for Vaccine Sciences, University of Texas Medical Branch at Galveston

Background: Seasonal influenza vaccination is recommended by the Advisory Committee on Immunization Practices for people 6 months of age or older, followed by a second dose $\geq 4$ weeks later for some children 6 months through 8 years of age. Though maternal characteristics are known to impact the uptake of childhood influenza vaccination, studies are limited.

Objective: Examine associations between maternal characteristics and childhood influenza vaccination.

Methods: Maternal demographic characteristics and immunization data from children ages 19-35 months who had adequate provider data $(n=15,657)$ in the 2018 National Immunization Survey-Child were used. Influenza vaccination was considered up to date if children received 2 or more doses at least 4 weeks and 4 days apart by the time the child was 24 months of age. Bivariate analyses, including chi-square tests with survey analysis settings, were conducted using Stata SE version 16.1 with $\alpha=0.05$.

Results: Over half $(56.1 \%)$ of children had 2 or more influenza vaccines by 24 months of age. There were differences in influenza vaccination among groups for maternal education (47.4\% for those with less than 12 years of education versus $69.1 \%$ for college graduates), age (45.9\% with mothers 29 years old or younger versus $61.6 \%$ with mothers 30 years old or older), and marital status $(62.7 \%$ for children of married mothers versus $45.3 \%$ for children of unmarried mothers). There were no differences between groups for survey language.

Conclusions: There were differences in childhood influenza vaccination between groups for most maternal characteristics. Though targeted interventions for families at risk for influenza undervaccination may be warranted, increasing influenza vaccination in all demographic groups remains imperative.

\section{School Staff Perceptions about the Impact of Physical Activity on Girls Versus Boys: A Cross-Sectional Study}

David O. Okanlawon, ${ }^{1}$ Maria E. Fernandez, ${ }^{1}$ Timothy J. Walker (presenting author) ${ }^{1}$

\section{${ }^{1}$ The University of Texas Health Science Center at Houston}

Background: Research indicates that girls are less physically active than boys during school. Because school staff members play a key role in providing physical activity opportunities, it is important to understand differences in staff perceptions about physical activity for boys as compared with girls.

Objective: Compare elementary school staff members' perceptions about the need for physical activity for boys versus girls.

Methods: We conducted a cross-sectional study with an urban school district in Texas. We surveyed staff from 20 elementary schools in the summer and fall of 2019. Participants were administrators, classroom teachers, physical education teachers, and support staff. The survey included questions about the consequences of not participating in adequate amounts of physical activity (using a 5-point Likert scale) and asked these 
questions in reference to boys and girls separately. We compared staff responses using mixed-effects linear regression models, controlling for respondents' gender, job type, job years, and age.

Results: A total of 205 people responded to the survey $(96 \%$ female, $66 \%$ classroom teachers, average job years $=6.6$, average age $=40$ years). Participants reported different expectations related to the negative effects of insufficient physical activity for boys versus girls. Specifically, with inadequate physical activity, staff perceived boys to (1) act up more in class (boys $=4.4$, girls $=3.3, P<0.001$ ), (2) have poor academic performance (boys $=4.2$, girls $=3.5, P<0.001$ ), (3) lose focus (boys $=4.2$, girls $=3.6, P<0.001$ ), and (4) not do well in school (boys $=4.0$, girls $=3.4, P<0.001$ ).

Conclusions: Results suggest that school staff members perceive boys to be impacted more by inadequate physical activity than they perceive girls to be, which may influence efforts to encourage and support physical activity across genders.

\section{University of Utah}

\section{Mechanisms of Preterm Infant Retinopathy Protection in Maternal Preeclampsia}

Kinsey Shirer, ${ }^{2}$ Blair Wood, ${ }^{1}$ Lara Carroll, ${ }^{1}$ Benjamin Haaland, ${ }^{3}$ Lakshmi D. Katikaneni, ${ }^{4}$ Margaret M. DeAngelis, ${ }^{1,5-6}$ Leah A. Owen (presenting author) ${ }^{1}$

${ }^{1}$ Department of Ophthalmology and Visual Sciences, University of Utah; ${ }^{2}$ Department of Ophthalmology, Medical University of South Carolina; ${ }^{3}$ Department of Population Health Sciences, University of Utah; ${ }^{4}$ Department of Pediatrics, Division of Neonatology, Medical University of South Carolina; ${ }^{5}$ Department of Pharmacotherapy, College of Pharmacy, University of Utah; ${ }^{6}$ Department of Ophthalmology, Jacobs School of Medicine and Biomedical Sciences, State University of New York at Buffalo

Background: Retinopathy of prematurity (ROP) is a blinding retinal vascular aberrancy in preterm infants. We cannot prevent or cure ROP blindness. A natural form of ROP protection exists in the setting of preeclampsia; greater understanding of the molecular basis for this protection paradigm may allow for development of disease prevention strategies. As ischemia is a central feature in both ROP and preeclampsia, we hypothesize that angiogenesis mediators facilitate ROP protection.

Methods: We analyzed peripheral expression of candidate proteins with suggested roles in preeclamptic and ROP pathophysiology and with a direct or indirect angiogenesis function, using ELISA (HTRA1, IGF1) or Luminex (TGF $\beta-1$ and VEGF$A$ ). Placental expression was assessed with immunohistochemistry and murine retinal expression using reverse transcriptase polymerase chain reaction (RT-PCR). Functional analysis was assessed in vivo using established murine models of ROP.

Results: We recruited maternal-infant pairs $(n=39)$ with and without preeclampsia and preterm infants (GA $<32$ weeks) $(\mathrm{n}=75)$ from 2 institutions. Only HTRAl expression was significantly associated with ROP and preeclampsia; specifically, elevated HTRAl directly correlated with ROP and inversely correlated with preeclampsia. Immunohistochemistry demonstrated that placental HTRA 1 expression was similarly increased in the absence of preeclampsia. RT-PCR demonstrated elevated murine HTRA1 retinal expression in ROP, and transgenic mice with elevated HTRA1 demonstrated greater ROP disease severity in the oxygen-induced retinopathy model. Finally, pretreatment with intravitreal anti-HTRAl antibody reduced ROP disease in this model.
Conclusions: We provide multiple lines of evidence to support a role for HTRAl in preeclampsia-mediated ROP protection, providing the first potential therapeutic target for ROP prevention within this paradigm.

\section{Gender Bias in Medicine Negatively Impacts Diagnosis and Clinical Care for Individuals with Complex Medical Conditions}

James Hemp, ${ }^{2}$ Douglas Ball, ${ }^{3}$ Michael Green, ${ }^{4}$ Bradley Jensen, ${ }^{4}$ Robert Shingleton, ${ }^{4}$ Stephen K. Trapp, ${ }^{4}$ Laura A. Pace (presenting author) ${ }^{1}$

${ }^{1}$ Center for Genomic Medicine, University of Utah;

${ }^{2}$ Department of Internal Medicine, University of Utah; ${ }^{3}$ Division of Pediatric Genomics, University of Utah; ${ }^{4}$ Division of Physical Medicine \& Rehabilitation, University of Utah

Background and Objectives: The Ehlers-Danlos syndromes (EDS) are rare genetic connective tissue disorders with sexdependent phenotypes. Affected females tend to have a more disabling clinical course and more severe manifestations of comorbid conditions, such as dysautonomia and immune-mediated disorders. Because of the complexity of EDS and comorbid conditions, coupled with gender bias against women within the US medical system, many patients are first diagnosed with psychiatric or functional disorders. This has led numerous major medical centers to refuse care for patients with a known or suspected EDS. To address this discriminatory practice, we created a multidisciplinary EDS clinic to aid in the diagnosis and treatment of patients with suspected EDS and associated comorbid conditions.

Methods: There were 65 patients with a suspected EDS who were seen in the Multidisciplinary EDS Clinic at the University of Utah. Patients underwent a 5-hour clinical evaluation with 5 practitioners (a clinical geneticist, neurogastroenterologist, clinical psychologist, physiatrist, and physical therapist), as well as genetic testing. Descriptive statistics were used to evaluate outcomes.

Results and Conclusions: We confirmed a diagnosis of an EDS in $57 \%$ of patients ( $32 \mathrm{hEDS}, 3 \mathrm{mEDS}, 2 \mathrm{aEDS}$ ). Of all the patients, $90 \%$ had concerning comorbid conditions, and $85 \%$ of patients who underwent autonomic testing were diagnosed with a dysautonomia. These results demonstrate that a thorough clinical evaluation is required in this population. Patients with EDS are wrongly dismissed by the medical community because of their medical complexity and the bias to diagnose women with psychological disorders rather than properly evaluate their symptoms.

\section{University of Wisconsin-Madison}

\section{The University of Wisconsin BIRCWH Program}

Elizabeth S. Burnside, ${ }^{1}$ Evie Carchman, ${ }^{2}$ Rebecca Myerson, ${ }^{3}$ Judith Simcox, ${ }^{4}$ Marsha Mailick ${ }^{5}$

${ }^{1}$ Department of Radiology, School of Medicine and Public Health, University of Wisconsin-Madison;

${ }^{2}$ Department of Surgery, School of Medicine and Public Health, University of Wisconsin-Madison;

${ }^{3}$ Department of Population Health Sciences, School of Medicine and Public Health, University of Wisconsin-Madison;

${ }^{4}$ Department of Biochemistry, College of Agricultural and Life Sciences, University of Wisconsin-Madison; ${ }^{5}$ Waisman Center, Office of the Vice Chancellor for Research and Graduate Education, University of Wisconsin-Madison 
Background: Consistently a high priority, interdisciplinary women's health research thrives at the University of Wisconsin (UW) because of the overall climate of excellence, stellar research career development programs, and dedication to collaboration among diverse disciplines.

Objective: To summarize the UW BIRCWH program's career development philosophy and introduce 3 outstanding Scholars whose science embodies the Trans-NIH Strategic Plan for Women's Health Research.

Approach/Scholars: The UW BIRCWH program fosters novel interdisciplinary collaborations with over 40 mentors from schools of medicine, nursing, pharmacy, engineering, veterinary medicine, and others. We build on already strong institutional career development and nationally renowned mentoring programs advanced by the UW Clinical and Translational Science Awards (CTSA) to train Scholars who will lead women's health research and accelerate translation to patients and communities. Our Scholars include Judith Simcox, PhD, an Assistant Professor in Biochemistry who will be discovering circulating lipid biomarkers for metabolic disease in African American women; Rebecca Myerson, $\mathrm{PhD}$, an Assistant Professor in Population Health Sciences who will be investigating the effect of health insurance policy and gender differences on cancer care and outcomes; and Evie Carchman, MD, an Assistant Professor in Surgery who will understand the use of protease inhibitors to prevent anogenital cancer in women. The UW program capitalizes on and strengthens institutional/leadership foundations in cancer, health disparities, and informatics/big data.

Innovation: The UW BIRCWH program will develop novel training methods to foster team science skills and an evidencebased career guidance model. Scholars will engage with curricular interventions to establish and test a career coaching model.

Conclusions: The UW BIRCWH program is poised to powerfully advance ORWH's strategic priorities.

\section{Vanderbilt University}

\section{Jo-1 Autoantigen-Specific B Cells Are Skewed Toward Distinct Functional B-cell Subsets in Patients with Antisynthetase Syndrome}

Jennifer Young-Glazer, ${ }^{1}$ Alberto Cisneros, ${ }^{1}$ Erin Wilfong, ${ }^{1,3}$ Leslie J. Crofford, ${ }^{1,2}$ Rachel H. Bonami (presenting author) ${ }^{1,2}$

${ }^{1}$ Division of Rheumatology and Immunology, Department of Medicine; ${ }^{2}$ Department of Pathology, Microbiology, and Immunology; ${ }^{3}$ Division of Allergy, Pulmonary, and Critical Care, Department of Medicine, Vanderbilt University Medical Center

Background: Antisynthetase syndrome (AS), a rare but severe systemic autoimmune disease, disproportionately affects females (2:1). Clinically diagnostic class-switched Jo-1 autoantibodies signal escape of Jo-1-specific B cells from normal immune checkpoints to create AS liability. It is not known how Jo-1-specific B cells escape immune tolerance to differentiate and expand in AS. Identifying which functional B-cell subset(s) contain Jo-1-specific B cells in AS is necessary to prevent immune tolerance breach and subsequent disease.

Objective: To test whether Jo-1-specific B cells differ in functional subset distribution compared with the broad B-cell pool in AS patients.

Methods: Flow cytometry was used to measure proportions of B-cell subsets stratified by Jo- 1 binding specificity in $10(80 \%$ female) Jo-1 autoantibody-positive AS patients compared with 8 (63\% female) age-matched healthy controls. Mann-Whitney U tests tested significance $(P<0.05)$.
Results: Among Jo-1-specific B cells, most $($ mean $=65 \%)$ were $\mathrm{IgM}+$ (not class-switched); autoreactive-prone CD21 ${ }^{\text {low }}$ and memory $\mathrm{B}$ cells were increased but antibody-secreting plasmablasts were decreased relative to non-Jo-1-specific B cells in the same donors and total B cells in healthy controls.

Conclusions: Limited class switching and differentiation to antibody-secreting cells suggests that some immune tolerance checkpoints are preserved for Jo-1-specific B cells in AS patients. However, Jo-1-specific B cells could propagate AS independently of autoantibody production by presenting autoantigen to T cells. Thus, pathologic Jo-1-specific B cells could be measured as an additional correlate for immune therapy responsiveness in AS and may help predict disease to allow earlier intervention.

\section{Sex Differences in Fatty Infiltration of Muscles in MRI-Confirmed Rotator Cuff Tears}

Thomas Freeman, ${ }^{3}$ John E. Kuhn, ${ }^{3}$ Nitin Jain, ${ }^{2,4,5}$ Ayush Giri (presenting author) ${ }^{1,2}$

${ }^{1}$ Division of Quantitative Sciences, Department of Obstetrics and Gynecology, Vanderbilt University Medical Center; ${ }^{2}$ Division of Epidemiology, Department of Medicine, Vanderbilt University Medical Center; ${ }^{3}$ Department of Orthopedic Surgery and Rehabilitation, Sports Medicine Center, Vanderbilt University Medical Center; ${ }^{4}$ Department of Physical Medicine and Rehabilitation, Vanderbilt University Medical Center; ${ }^{5}$ Departments of Physical Medicine and Rehabilitation, Orthopedics, and Population and Data Sciences, University of Texas Southwestern

Background: Fatty infiltration of the rotator cuff musculature is associated with poor recovery outcomes in patients who have had surgical repair for a rotator cuff tear. Sex differences in short-term postsurgical recovery indices are also observed. Whether fatty infiltration in the rotator cuff musculature differs by sex is unknown.

Objective: To evaluate whether presence of fatty infiltration differs by sex in magnetic resonance imaging (MRI)-confirmed rotator cuff tears.

Methods: We used data from the Rotator Cuff Outcomes Workgroup (ROW) cohort and the Multicenter Orthopaedic Outcomes Network (MOON) Shoulder Group cohort with confirmed cuff tears and available MRI images. Cuff muscle fatty infiltration was scored from MRIs using the Goutallier system (0: no infiltration; $1-4$ : increasing severity of infiltration) and dichotomized as absent or present for analysis. We used logistic regression to evaluate the relationship between sex and fatty infiltration, adjusting for age, body mass index, tear size, and tear duration. Cohorts were analyzed separately and metaanalyzed using inverse variance weights to present odds ratios (ORs) and 95\% confidence intervals (CIs).

Results: There were 57 individuals $(36 \%)$ in the ROW cohort and $27(34 \%)$ in the MOON Shoulder Group cohort who had fatty infiltration in the rotator cuff. Of those with tears, women were 4.5 times as likely as men to have fatty infiltration in the cuff (meta-analysis $\mathrm{OR}=4.5 ; 95 \% \mathrm{CI}=1.9,10.4$ ). These associations were consistent across independent cohorts: ROW $\mathrm{OR}=3.2(95 \% \mathrm{CI}=1.1,9.4) ; \mathrm{MOON} \mathrm{OR}=7.5(95 \% \mathrm{CI}=2.0$, 28.7).

Conclusions: In rotator cuff tears, women were more likely to have fatty infiltration of the involved musculature than men. Whether this difference mediates the association between sex and short-term postsurgical recovery indices warrants investigation. 


\section{Sex-stratified Genome-Wide Association Study of Estimated Glomerular Filtration Rate Identifies Loci with Sex-Specific Effects on Kidney Function}

Eric S. Torstenson, ${ }^{2,3,5}$ Todd L. Edwards, ${ }^{2,3,5}$

Digna R. Velez Edwards, 2,3,6,7 Jacklyn N. Hellwege (presenting author) ${ }^{1-4}$

${ }^{1}$ Division of Genetic Medicine, Department of Medicine, Vanderbilt University Medical Center; ${ }^{2}$ Vanderbilt Genetics Institute, Vanderbilt University; ${ }^{3}$ Vanderbilt Epidemiology Center, Vanderbilt University Medical Center; ${ }^{4}$ Vanderbilt Center for Kidney Disease, Vanderbilt University Medical Center; ${ }^{5}$ Division of Epidemiology, Department of Medicine, Vanderbilt University Medical Center; ${ }^{6}$ Division of Quantitative Sciences, Department of Obstetrics and Gynecology, Vanderbilt University Medical Center; ${ }^{7}$ Department of Biomedical Informatics, Vanderbilt University Medical Center

Background: Estimated glomerular filtration rate (eGFR) is a measure of kidney function. Reduced eGFR defines renal dysfunction and stages of chronic kidney disease (CKD). We need to understand biological mechanisms contributing to reduced eGFR and CKD so treatments targeting specific biological factors can be developed.

Objective: Identify genetic loci with differential associations with kidney function through a sex-stratified genome-wide association study of eGFR, followed by association with predicted gene expression in kidneys.

Methods: We have identified self-reported blacks $(n=7,919$ females and 5,482 males) and whites $(n=38,239$ females and 30,026 males) with clinically assessed serum creatinine measurements and genetic data in Vanderbilt's BioVU resource. eGFR was regressed onto over 16 million high-quality genetic variants for evaluation of additively encoded genetic variants, age, body mass index, and 10 principal components, followed by inverse varianceweighted fixed-effects meta-analyses within and across races.

Results: We identified 34 significant single-nucleotide polymorphisms (SNPs) at the APOL1 locus, with consistent effects across sexes in blacks, though effects were larger and more significant in males. There were 6 SNPs (4 loci) outside of the APOL1 locus that were associated in males only, 4 of which were consistent in direction with females. There were 22 SNPs (5 loci) associated in females only, and 7 of them were in consistent direction with males.

Conclusions: This work suggests that there may be genes that impact eGFR levels differentially by sex. Future directions will use predicted gene expression analysis to localize sex-specific associations to genes and evaluate these genes as targets of existing therapeutics.

\section{Type 2 Nasal Immune Responses to Respiratory Syncytial Virus in Female Infants Are Associated with Later Childhood Recurrent Wheeze}

Tebeb Gebretsadik, ${ }^{2}$ Derek A. Wiggins, ${ }^{1}$ Christian Rosas-Salazar, ${ }^{3}$ Dawn Newcomb, ${ }^{1}$ Larry J. Anderson, ${ }^{4}$ Tina V. Hartert, ${ }^{1}$ Kedir N. Turi (presenting author) ${ }^{1}$

${ }^{1}$ Department of Medicine, Vanderbilt University School of Medicine; ${ }^{2}$ Department of Biostatistics, Vanderbilt University School of Medicine; ${ }^{3}$ Department of Pediatrics, Vanderbilt University School of Medicine; ${ }^{4}$ Department of Pediatrics, Emory University School of Medicine
Background: The incidence of recurrent wheeze in early childhood is higher in boys than in girls; however, the reasons are not understood. Wheeze pathogenesis may be sex-specific.

Objective: To identify sex-stratified immune-response patterns during respiratory syncytial virus (RSV) infection and assess associations with recurrent wheezing.

Methods: Nasal wash samples collected during acute RSV infant infection from 298 (44\% female) infants in the INSPIRE birth cohort were assayed for 53 cytokines, chemokines, and growth factors using the Luminex multiplex bead-based assays. Children were assessed for recurrent wheezing (3 or more wheezing episodes) yearly for 4 years using the International Study of Asthma and Allergies in Childhood questionnaire. We used a sex-stratified unsupervised clustering approach to identify immune-response patterns. We used generalized estimating equations to assess associations between the identified sexstratified clusters and 1-, 2-, 3-, and 4-year recurrent wheezing, adjusted for covariates.

Results: Three distinct immune clusters were identified and characterized as follows: Cluster-1, type 2 (T2) immune responses (both sexes: TSLP, IL-23, IL-33); Cluster-2, type 1 (T1) immune responses (males: MCP-2, MIP-1d, IL-2) and T2 immune responses (females: IL-5, IL-4, IL-2); and Cluster-3, T1 immune responses (both sexes: IL-12, EGF, GC-SF). In females, Cluster-1 $(\mathrm{aOR}=8.78,95 \% \mathrm{CI}=[1.55,49.71])$ and Cluster-2 $(\mathrm{aOR}=8.97,95 \% \mathrm{CI}=1.49,54.03)$ were associated with higher odds of recurrent wheezing than Cluster-3. In males, Cluster-1 $(\mathrm{aOR}=1.18,95 \% \mathrm{CI}=0.52,2.68)$ and Cluster $-2(\mathrm{aOR}=1.53$, $95 \% \mathrm{CI}=0.71,3.27)$ were not significantly associated with recurrent wheezing.

Conclusions: Female infants with T2-dominated immune responses to RSV infection were at increased odds of childhood recurrent wheezing; this was not observed in males.

\section{Sex-Specific Risk of Opioid-Related Overdose and Death}

Sarah S. Osmundson, ${ }^{2}$ Margaret Adgent, ${ }^{1}$ Ed Mitchel Jr., ${ }^{1}$ Carlos G. Grijalva, ${ }^{1,3}$ Andrew D. Wiese (presenting author) ${ }^{1}$

${ }^{1}$ Department of Health Policy, Vanderbilt University Medical Center; ${ }^{2}$ Department of Obstetrics and Gynecology, Vanderbilt University Medical Center; ${ }^{3}$ Mid-South Geriatric Research Education and Clinical Center, VA Tennessee Valley Healthcare System

Background: Opioid-related overdoses increased substantially during the past decade, and some studies suggest a higher increase was observed among women than among men. However, few studies have comprehensively accounted for the complex interplay of clinical, societal, and biological differences between men and women to determine whether the risk of overdose varies by sex of the opioid user.

Objective: To test the hypothesis that opioid-naïve women initiating use of prescribed opioids have a higher incidence of overdose and opioid-related death than men.

Methods: We will construct a retrospective cohort of opioidnaïve patients enrolled in Tennessee Medicaid (TennCare) who initiated use of opioid analgesics (2006-2017). We will supplement TennCare data with Medicare Part D pharmacy data and a statewide hospitalization and death registry to characterize prescription opioid use and outcomes during follow-up. We will use marginal structural Cox proportional hazard regression models to determine the causal effect of sex on the risk of 
overdose and death, accounting for fixed and time-varying confounding assessed at baseline and monthly thereafter during follow-up. In addition to reporting the association between sex and the risk of overdose and death, we will quantify the role of mediator factors in the observed associations, including prescription characteristics, substance use disorder treatment, and co-occurring psychiatric disorders.

Results: We identified more than 530,000 opioid-naïve patients initiating opioid use in a preliminary cohort of adult TennCare patients (about $60 \%$ female) that will be used to examine the incidence of overdose and opioid-related death among women compared with men.

Conclusions: Characterizing sex differences in the risk of overdose at the time of opioid initiation will assist in the development of sex-specific prevention approaches.

\section{Sex-Specific Effect of Cholesteryl Ester Transfer Protein in Murine HDL Function}

Christopher H. Emfinger, ${ }^{2}$ Kelly Fahey, ${ }^{3}$ Lin Zhu, ${ }^{1,8}$ Siwei Zhang, ${ }^{6}$ Yaomin Xu, ${ }^{7}$ John M. Stafford, ${ }^{1,4,5}$ Sophia S. Yu (presenting author) ${ }^{1}$

${ }^{1}$ Division of Diabetes, Endocrinology, and Metabolism, Department of Internal Medicine, Vanderbilt University Medical Center; ${ }^{2}$ Department of Biochemistry, University of Wisconsin; ${ }^{3}$ Oakland University William Beaumont School of Medicine; ${ }^{4}$ VA Tennessee Valley Healthcare System; ${ }^{5}$ Department of Molecular Physiology and Biophysics, ${ }^{6}$ Department of Biomedical Information, ${ }^{7}$ Department of Biostatistics, Vanderbilt University School of Medicine
Background: High-density lipoprotein (HDL) particles attenuate atherosclerotic cardiovascular disease (ASCVD) risk, but there may be sex differences in HDL's protective effect. Cholesteryl ester transfer protein (CETP) is a lipid transfer protein that shuttles lipids between HDL and other lipoproteins and contributes to HDL levels and functions. We hypothesize that expression of CETP in HDL function underpins sex differences in ASCVD risk in humans.

Objective: As mice do not naturally express CETP, we use CETP transgenic mice that have a human-like lipid profile and wild-type (WT) mice to investigate the role of CETP in malefemale differences in key HDL functions of anti-inflammation and antioxidation.

Methods: Eight-week-old male and female WT and CETP transgenic mice were sacrificed after a 5-hour fast. HDL was isolated via apolipoprotein B precipitation. We measured HDL's antioxidative and anti-inflammatory functions ex vivo. Groups were compared with Student's t-test $(P<0.05)$.

Results: In WT mice, HDL's antioxidation and antiinflammation functions did not differ by sex. However, in CETP transgenic mice, HDL from females had worse antioxidative function $(P<0.05)$ and less ability to suppress the VCAM-1 marker of inflammation $(P<0.001)$ than HDL from males.

Conclusions: Previous clinical trials inhibiting CETP raised HDL levels but had a neutral to negative impact on ASCVD. Conversely, our findings suggest that CETP contributes to a less atheroprotective HDL in female mice. Next steps include gonadectomizing mice and reintroducing estradiol and testosterone in different chromosomal sex backgrounds to determine whether sex hormones or sex chromosomes contribute to CETP's effect on HDL function. 